\title{
Quasisteady quasihomogeneous description of the scale interactions in near-wall turbulence
}

\author{
Chi Zhang* and Sergei I. Chernyshenko ${ }^{\dagger}$ \\ Department of Aeronautics, Imperial College London, South Kensington, \\ London SW7 2AZ, United Kingdom \\ (Received 12 January 2016; published 25 May 2016)
}

\begin{abstract}
By introducing the notion of an ideal large-scale filter, a formal statement is given of the hypothesis of the quasisteady quasihomogeneous nature of the interaction between the large and small scales in the near-wall part of turbulent flows. This makes the derivations easier and more rigorous. A method is proposed to find the optimal large-scale filter by multiobjective optimization, with the first objective being a large correlation between largescale fluctuations near the wall and in the layer at a certain finite distance from the wall and the second objective being a small correlation between the small scales in the same layers. The filter is demonstrated to give good results. Within the quasisteady quasihomogeneous theory, expansions for various quantities are found with respect to the amplitude of the largescale fluctuations. Including the higher-order terms improves the agreement with numerical data. Interestingly, it turns out that the quasisteady quasihomogeneous theory implies a dependence of the mean profile logarithmic-law constants on the Reynolds number. The main overall result of the present work is the demonstration of the relevance of the quasisteady quasihomogeneous theory for near-wall turbulent flows.
\end{abstract}

DOI: 10.1103/PhysRevFluids.1.014401

\section{INTRODUCTION}

First clear observations and specific studies of the relationships between the large-scale and small-scale motions in near-wall turbulence appeared in the 1970s [1-3]. Recent reviews [4-6] provide a good introduction to the current state of the art. This topic is growing in importance with continuing [7] accumulation of evidence of the second peak (or plateau) in turbulence energy distribution at high Reynolds numbers Re. Due to the well-known limitations, both direct numerical simulations and wind-tunnel experiments on near-wall turbulence have to be done at values of Re well below the values of Re characteristic of major aerospace and marine applications. This necessitates extrapolating the results to large Re. Prior to the discovery of the second peak and outer-inner coupling the extrapolation was thought to be justified by the universality of near-wall turbulence. Universality means that if expressed in wall units that are units based on the mean friction at the wall, the statistical characteristics of the near-wall turbulence are virtually independent of Re. This implies independence of other factors, such as the external pressure gradient or the wall curvature, which, if expressed in wall units, depend on Re but tend to either zero or infinity as $\operatorname{Re} \rightarrow \infty$. However, the coupling of large and small scales means that the near-wall turbulence is not universal in the above sense and the existence of the second, outer, peak in turbulence energy, emerging as Re increases, means that even if the outer-inner interaction is not quantitatively large in direct numerical simulations and wind-tunnel experiments, it might be large or even dominant in the flows encountered in practice. Hence, ways of extrapolating to large Re need to be found without relying on the classical universality hypothesis.

While the present study is of general nature, focusing our attention on the near-wall region and our choice of the numerical data source were motivated by the particular need for such an extrapolation

\footnotetext{
*c.zhang13@imperial.ac.uk

${ }^{\dagger}$ s.chernyshenko@imperial.ac.uk
} 
tool in the area of turbulent skin friction reduction. The state of the art of drag reduction as of 2010 is comprehensively covered in Ref. [8]. In the part related to the present study it can be summarized in the following way. The only so-far-known practically feasible method of turbulent friction reduction is riblets [9]. However, taking into account the Reynolds number effects using the classical universality hypothesis, as proposed by [10], shows [11] that the effectiveness of riblets is not sufficient for applications in aeronautics. The known active methods, involving suction and blowing through the wall [12], plasma actuation [13], and in-plane wall motion, are nominally more efficient [14]. However, the complexity of the practical implementation of these known active methods is too large [11]. Developing alternative, equally effective but practically feasible methods of drag reduction requires theory yielding to intuitive understanding. In the years following 2010 such theories appeared, making it possible to approximately predict important organized structures (streaks) present in the flow in the regime with drag reduction [15] and the drag reduction itself [16,17] and proposals of practically feasible methods of drag reduction started to emerge [18]. However, all these results are verified only by comparisons with direct numerical simulations conducted at moderate values of Re. From the hypothesis of the universality of near-wall turbulence it follows that as Re increases the drag reduction should decrease slowly [10], but direct numerical simulations [19] appear to indicate a noticeably faster decrease. A mechanism by which coupling of large and small scales can lead to this faster decrease was proposed [20], but no quantitative analysis was done. Overall, the state of the art in drag reduction studies requires the issue of extrapolating to large $\mathrm{Re}$ to be clarified. The research described in the present paper is a step in this direction.

A particular way of extrapolation to large Re was implied by a series of studies by Marusic and co-workers [21-25]. The basic setup of their work involves two probes measuring simultaneously the velocity at two points in the flow, the wall probe located closer to the wall than the outer probe. The signal obtained from each probe is then filtered so that the velocity can be represented as a sum of a large-scale component and a small-scale component. Then the relationship

$$
u^{\prime+}\left(y^{+}\right)=\alpha\left(y^{+}\right) u_{L}^{\prime+}\left(y_{\mathrm{o}}^{+}\right)+\left[1+\beta\left(y^{+}\right) u_{L}^{\prime+}\left(y_{\mathrm{o}}^{+}\right)\right] u_{\mathrm{u}}^{\prime+}\left(y^{+}\right)
$$

is introduced, where $u^{\prime+}$ is the velocity fluctuation, $u_{L}^{\prime+}$ is its large-scale component, $y^{+}$is the distance from the wall to the wall probe, and $y_{0}^{+}$is the distance from the wall to the outer probe. The quantities expressed in wall units are marked with a plus superscript and all fluctuations, that is, the deviations from the average, are marked with primes. The functions $u^{\prime+}, u_{L}^{\prime+}$, and $u_{\mathrm{u}}^{\prime+}$ depend also on time and the other two coordinates omitted for brevity. The basic empirical observation is that it is possible to find such universal, that is, Re-independent, functions $\alpha\left(y^{+}\right)$and $\beta\left(y^{+}\right)$and such a dependence of $y_{\mathrm{o}}^{+}$on Re that the statistical characteristics of the universal velocity field $u_{\mathrm{u}}^{\prime+}$ defined by (1) are independent of Re.

If this observation is correct, it resolves the issue of extrapolating to large Re. For example, one can perform a direct numerical simulation of the flow in the drag-reducing regime at a moderate $\mathrm{Re}$ and determine the statistical properties of the corresponding $u_{\mathrm{u}}^{\prime+}$ from (1). Then, in a physical experiment at high $\operatorname{Re}$ the statistical properties of $u_{L}^{\prime+}\left(y_{\mathrm{o}}^{+}\right)$can be measured. This is easier than measuring the statistical properties of $u^{\prime+}\left(y^{+}\right)$, since the outer probe is located much farther away from the wall and the large-scale component has a lower frequency range. Then the statistical properties of $u^{\prime+}(y)$ at this high Re can be recovered from (1).

Formula (1) was found to be reasonably accurate, but more detailed studies [24,26] showed that the difference of the response of the near-wall turbulence to positive and negative large-scale fluctuations of the same magnitude is not in full agreement with the implications of (1). Also, the empirical relation (1) was verified only against cases in which the full signal from both probes was available. Hence, extrapolating to Re so high that the signal from the near-wall probe cannot be obtained still requires a leap of faith. A theoretical justification for (1) would increase the level of confidence in its validity and allow refining it.

Chernyshenko et al. [27] derived (1) from the hypothesis that the effect of the large-scale motion on the small-scale motion is of quasisteady nature, combined with the assumption that the amplitude of the large-scale fluctuations is small, so that linearization is possible. The rigorous 


\section{QUASISTEADY QUASIHOMOGENEOUS DESCRIPTION OF ...}

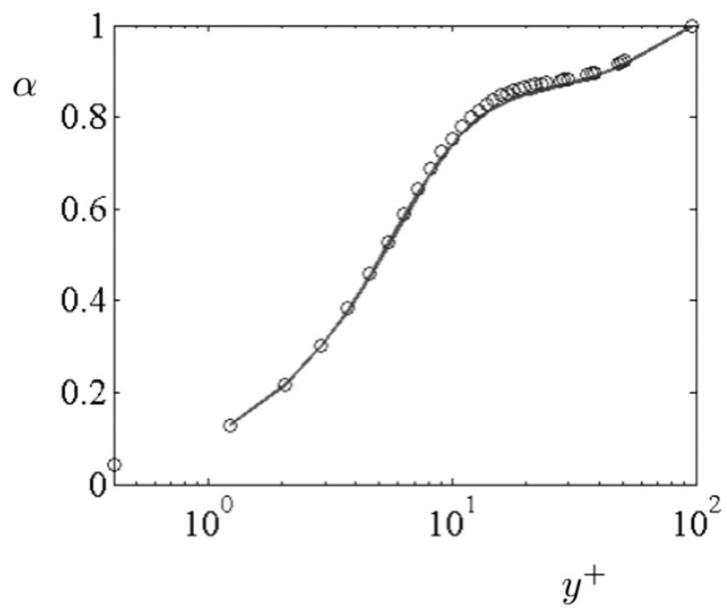

FIG. 1. Symbols represent $\alpha=\left\langle u_{L}^{\prime}\left(y^{+}\right) u_{L}^{\prime}\left(y_{0}^{+}\right)\right\rangle /\left\langle u_{L}^{\prime+2}\left(y_{0}^{2}\right)\right\rangle$, which is the superposition coefficient first introduced in Refs. [21-25] [see (1)], and the curve shows $\left[U\left(y^{+}\right)+y^{+} d U\left(y^{+}\right) / d y^{+}\right] /\left[U\left(y_{0}^{+}\right)+\right.$ $\left.y_{\mathrm{o}}^{+} d U\left(y_{\mathrm{o}}^{+}\right) / d y_{\mathrm{o}}^{+}\right]$. Both quantities were obtained from direct numerical simulations (full details are in Sec. IV). The theory in the following sections explains why these quantities almost coincide.

formal statement of the quasisteady hypothesis is given in Sec. II. From (1) it follows that $\alpha\left(y^{+}\right)=\left\langle u_{L}^{\prime}\left(y^{+}\right) u_{L}^{\prime}\left(y_{0}^{+}\right)\right\rangle /\left\langle u_{L}^{\prime+2}\left(y_{\mathrm{o}}^{2}\right)\right\rangle$, where the angular brackets denote averaging. In Ref. [27] it was derived that $\alpha\left(y^{+}\right)$should be approximately equal to $\left[U\left(y^{+}\right)+y^{+} d U\left(y^{+}\right) / d y^{+}\right] /\left[U\left(y_{0}^{+}\right)+\right.$ $\left.y_{\mathrm{o}}^{+} d U\left(y_{\mathrm{o}}^{+}\right) / d y_{\mathrm{o}}^{+}\right]$, where $U\left(y^{+}\right)$is the mean velocity profile. Figure 1 shows that the predicted equality of these two seemingly unrelated quantities is satisfied with remarkable accuracy. Note that Fig. 1 is obtained in the present study with a better large-scale filter than the filter used in Ref. [27] and that the agreement is in part fortuitous. The superposition coefficient $\alpha\left(y^{+}\right)$introduced in Refs. [21-25] is defined in such a way that its value is exactly zero at the wall and exactly 1 at $y^{+}=y_{\mathrm{o}}^{+}$. Hence, any reasonable theory, whether it is correct or not, would give the same values at these points. In the following sections many more comparisons will be made. For $\operatorname{Re}_{\tau}=1000$ the typical discrepancy will be roughly in the range of $10 \%$ and it can be expected to decrease as $\operatorname{Re}_{\tau}$ increases.

The quasisteadiness hypothesis is natural, since it can be expected that the characteristic time scale of the large-scale component of the velocity field is large compared to the characteristic time scale of the small-scale component of the velocity field. The validity of the quasisteadiness hypothesis was discussed on the basis of numerical [6] and experimental [24] results. The analysis of the phase shift between various properties of the large-scale and small-scale fluctuations, done in these works, is particularly illuminating. This analysis supports, at least approximately, the quasisteady hypothesis only in the area close enough to the wall. For example, the frequency modulation of small scales by large scales, predicted by the quasisteady hypothesis, is observed only for $y^{+}<100$ [24]. Fortunately, the drag-reduction mechanism is concentrated in the area below 100 wall units $[14,19,26]$. Measuring the large-scale fluctuations at $y^{+}=100$ for the values of Re typical for aeronautical applications is a realistic task. Given the particular motivation of this paper, we will concentrate our attention on the near-wall region.

Linearizing with respect to the small amplitude of the large-scale fluctuations is convenient for analytic derivations, but it introduces an error. Quantifying this error is one of the goals of the present study. It will be achieved by calculating the higher-order terms of the expansion in the amplitude of large-scale perturbation.

In Ref. [27] the derivations were based on the intuitive idea that the large scales are much larger than the small scales and were, in this respect, not fully rigorous. This in itself is acceptable when 
dealing with the quasisteady hypothesis, which is only approximate. However, when derivations become cumbersome, as it is the case with the higher-order terms, relying on intuitive notions is difficult. The second goal of the present work is to introduce a rigorous framework making derivations easier. This will be achieved by formally postulating the necessary and sufficient properties of the filter.

The third goal of the present study is to propose a method of selecting a filter most suitable for application with the quasisteady hypothesis. To this end we will consider a certain multiobjective optimization problem.

Achieving these goals requires a fair amount of technical work. The reader more interested in the physical mechanisms might look through Secs. II A and II B for the basic assumptions and then go directly to Sec. V and the following sections.

\section{MAIN HYPOTHESES}

We separate the physical assumptions of this section from the rigorous mathematics that follows.

\section{A. Quasisteady quasihomogeneous hypothesis and the universality hypothesis}

The present analysis is restricted to flows statistically stationary in time and statistically homogeneous in the wall-parallel directions, such as a channel flow. Then the mean quantities depend only on the wall-normal coordinate. Let $t^{*}$ denote time, $y^{*}$ denote the wall-normal coordinate, and $x^{*}$ and $z^{*}$ denote the two remaining coordinates, $x^{*}$ being in the direction of the mean skin friction. Let $u^{*}\left(t^{*}, x^{*}, y^{*}, z^{*}\right)$ denote the $x$ component of the velocity. The asterisk superscript marks dimensional quantities. Within this paper the attention is limited to the properties of $u^{*}\left(t^{*}, x^{*}, y^{*}, z^{*}\right)$.

According to the classical view on the universality of near-wall turbulence, near the wall and at sufficiently large Re all statistical characteristics of the velocity field are independent of Re provided that all the variables are in wall units. This is not true if the influence of the outer structures does not tend to zero as $\operatorname{Re} \rightarrow \infty$. Hence, an alternative is required. We will now present in a refined, more rigorous, form the alternative assumption proposed in Ref. [27].

We assume that a large-scale filter is defined, that is, an operator with certain properties to be described in Sec. II B. Applied to any function depending on $t^{*}, x^{*}, y^{*}$, and $z^{*}$, this operator gives another function of the same arguments, which we will call a large-scale component. The large-scale components will be denoted by a subscript $L$. In particular, $\tau_{L}^{*}$ denotes the large-scale component of the skin friction $\tau^{*}$. Let

$$
u_{\tau_{L}}^{*}\left(t^{*}, x^{*}, z^{*}\right)=\sqrt{\tau_{L}^{*}\left(t^{*}, x^{*}, z^{*}\right) / \rho^{*}}
$$

where $\rho^{*}$ is the fluid density. Let

$$
\tilde{u}\left(\frac{t^{*} u_{\tau_{L}}^{* 2}}{v^{*}}, \frac{x^{*} u_{\tau_{L}}^{*}}{v^{*}}, \frac{y^{*} u_{\tau_{L}}^{*}}{v^{*}}, \frac{z^{*} u_{\tau_{L}}^{*}}{v^{*}}\right)=\frac{u^{*}\left(t^{*}, x^{*}, y^{*}, z^{*}\right)}{u_{\tau_{L}}^{*}\left(t^{*}, x^{*}, z^{*}\right)} .
$$

Here all the quantities are total, not fluctuations, that is, their mean values are not necessarily zero and $v^{*}$ is the kinematic viscosity.

We replace the classical universality hypothesis with the hypothesis that near the wall at sufficiently high $\operatorname{Re}$ the statistical characteristics of $\tilde{u}(\tilde{t}, \tilde{x}, \tilde{y}, \tilde{z})$ for constant $\tilde{t}, \tilde{x}, \tilde{y}$, and $\tilde{z}$ are independent of $\operatorname{Re}$, so $\operatorname{Re}$ affects only the statistical properties of $u_{\tau_{L}}^{*}\left(t^{*}, x^{*}, z^{*}\right)$. Note that if $u_{\tau_{L}}^{*}\left(t^{*}, x^{*}, z^{*}\right)$ is replaced with the mean friction velocity then (3) becomes the classical universality statement. Hence, the physical meaning of the proposed hypothesis is that rescaling should be done with large-scale-filtered skin friction rather than the mean skin friction. Then $u_{\tau_{L}}^{*}\left(t^{*}, x^{*}, z^{*}\right)$ and $\tilde{u}(\tilde{t}, \tilde{x}, \tilde{y}, \tilde{z})$ are expected to be statistically independent because the variation of the large-scale components with $t^{*}, x^{*}$, and $z^{*}$ is slow. Accordingly, this hypothesis will be called the quasisteady quasihomogeneous $(\mathrm{QSQH})$ universality hypothesis and the corresponding body of results that can be derived from it will be called the QSQH universality theory. 
Nondimensional variables based on $v^{*}$ and $\left\langle u_{\tau_{L}}^{*}\right\rangle$ will be used. This is similar to wall units, but with $\left\langle u_{\tau_{L}}^{*}\right\rangle$ instead of the dynamic velocity. In particular,

$$
u=\frac{u^{*}}{\left\langle u_{\tau_{L}}^{*}\right\rangle}, \quad u_{\tau_{L}}=\frac{u_{\tau_{L}}^{*}}{\left\langle u_{\tau_{L}}^{*}\right\rangle}, \quad t=\frac{t^{*}\left|u_{\tau_{L}}^{*}\right\rangle^{2}}{v^{*}}, \quad(x, y, z)=\left(x^{*}, y^{*}, z^{*}\right) \frac{\left\langle u_{\tau_{L}}^{*}\right\rangle}{v^{*}} .
$$

In these variables the QSQH hypothesis (3) can be written as

$$
u(t, x, y, z)=u_{\tau_{L}}(t, x, z) \tilde{u}\left(t u_{\tau_{L}}^{2}, x u_{\tau_{L}}, y u_{\tau_{L}}, z u_{\tau_{L}}\right) .
$$

Since the statistical properties of $u_{\tau_{L}}(t, x, z)$ depend on Re and the statistical properties of $\tilde{u}(\tilde{t}, \tilde{x}, \tilde{y}, \tilde{z})$ do not depend on $\mathrm{Re}$, these quantities have to be statistically independent. Note our use of mute variables, $\tilde{t}, \tilde{x}, \tilde{y}, \tilde{z}$, in this statement. In contrast, $u_{\tau_{L}}(t, x, z)$ and $\tilde{u}\left(t u_{\tau_{L}}^{2}, x u_{\tau_{L}}, y u_{\tau_{L}}, z u_{\tau_{L}}\right)$ with constant $t, x, y$, and $z$ are statistically dependent, the dependence entering via the arguments of $\tilde{u}$. This feature is the main source of interesting relationships to be derived. Statistical independence is understood here in the standard sense. In particular, the joint probability density function of the statistically independent variables is equal to the product of their marginal probability density functions.

The QSQH universality hypothesis can be subdivided in two. The first hypothesis is the statistical independence of $u_{\tau_{L}}(t, x, z)$ and $\tilde{u}(\tilde{t}, \tilde{x}, \tilde{y}, \tilde{z})$ at each particular Re. This can be called the QSQH hypothesis per se. The second hypothesis is that the statistical properties of $\tilde{u}(\tilde{t}, \tilde{x}, \tilde{y}, \tilde{z})$ are independent of Re. This can be called the hypothesis of QSQH Re-universality.

For very large values of $t, x$, and $z$ the scaled variables might respond strongly to the fluctuations of $u_{\tau_{L}}$ in the following sense. Since the amplitude of the fluctuations of $u_{\tau_{L}}$ is independent of $t$, the amplitude of fluctuations of the argument $t u_{\tau_{L}}^{2}$ of $\tilde{u}$ in Eq. (5) tends to infinity as $t$ tends to infinity. Therefore, for sufficiently large $t$ the amplitude of the fluctuation of $t u_{\tau_{L}}^{2}$ will become comparable or even much bigger than the characteristic time scale of the fluctuations. Then the assumptions underlying our theory will become inconsistent. Where the analysis involving large values of $t, x$, and $z$ is needed, the form of $\tilde{u}$ in Eq. (5) should be assumed in the spirit of the classic asymptotic theories for multiple-variable expansions [28], that is along the lines of replacing the definition of $t$, $x$, and $z$ given by (4) with $\partial t / \partial t^{*}=\left\langle u_{\tau_{L}}^{*}\right\rangle^{2} / \nu^{*}, \partial x / \partial x^{*}=\partial z / \partial z^{*}=\left\langle u_{\tau_{L}}^{*}\right\rangle / \nu^{*}$. Also, both $u$ and $u_{\tau_{L}}$ should actually be considered as vector quantities and the hypothesis should be refined accordingly, but we will not go further into these issues, as the specific comparisons we will make do not require this.

\section{B. Ideal large-scale filter properties}

To complete the formal introduction of the QSQH hypothesis, the properties of the large-scale filter $\mathcal{L}$ should be defined. Applied to any function, this filter generates another function of the same variables: $f_{L}(t, x, y, z)=\mathcal{L} f(t, x, y, z)$. The following properties are postulated.

(i) Linearity. $\mathcal{L}(a f+b g)=a \mathcal{L} f+b \mathcal{L} g$ for any $a, b, f(t, x, y, z)$, and $g(t, x, y, z)$.

(ii) Invariance of averages. The averaged variables are large scale: $\mathcal{L}\langle f\rangle=\langle f\rangle$.

(iii) Projection property. The large-scale filter does not change an already large-scale-filtered function: $\mathcal{L} \mathcal{L} f=\mathcal{L} f$.

(iv) Commuting with averaging. $\langle\mathcal{L} f\rangle=\mathcal{L}\langle f\rangle=\langle f\rangle$. The last of these two equalities is in fact property (ii), but we add it here because of the frequent use of these two properties together in our derivations, in particular in the form $\langle f\rangle=\langle\mathcal{L} f\rangle \equiv\left\langle f_{L}\right\rangle$.

(v) Scale-separation property. Applying the large-scale filter to any function of $t, x, y, z$, and other arguments that are large-scale-filtered variables is equivalent to averaging over the homogeneous directions and/or time $t, x$, and $z$ with the other arguments held constant:

$$
\mathcal{L} f\left(t, x, y, z, \mathcal{L} g_{1}, \ldots, \mathcal{L} g_{n}\right)=\left\langle f\left(t, x, y, z, \xi_{1}, \ldots, \xi\right)\right\rangle_{\xi_{1}=\mathcal{L} g_{1}, \ldots, \xi_{n}=\mathcal{L} g_{n}} .
$$


Finding a filter satisfying properties (i)-(iv) is possible. A cutoff in the Fourier space is an example. Property (v) is intimately related to the question of representing the flow field as a sum of large-scale and small-scale motions, that is, to the question of defining rigorously what large-scale motions are, which is a big challenge. For functions having a two-scale structure, such as $f(t, \epsilon t)$ with $\epsilon \ll 1$, it is easy to verify that averaging over an intermediate interval, that is, taking

$$
\mathcal{L} f(t, \epsilon t)=\frac{1}{2 T} \int_{t-T}^{t+T} f(\tilde{t}, \epsilon \tilde{t}) d \tilde{t}, \quad 1 \ll T \ll 1 / \epsilon,
$$

satisfies asymptotically all the required properties. This shows that the postulated properties of the filter are not self-contradictory. This also indicates that property (v) implies that in a certain sense the filter is equivalent to averaging over an interval or volume large compared to small scales but small compared to large scales. Turbulent flow fields, however, have multiscale structure and the question of finding a filter having all the required properties at least asymptotically as Re tends to infinity for turbulent flow fields remains open. One can, however, hope that there are filters that meet these requirements approximately. The stated filter properties will be used for rigorous derivations and then a filter will be chosen and comparisons made.

\section{QUASISTEADY QUASIHOMOGENEOUS THEORY}

Suppose now that a function $u(t, x, y, z)$ does have a form of (5), the operator $\mathcal{L}$ does have properties (i)-(v), and $u_{\tau_{L}}(t, x, z)$ is a large-scale-filtered quantity with a unit average. Then, regardless of the physical nature of these variables and the filter, a number of statements can be rigorously proved. This is the main goal of the present section. It is beneficial to clearly distinguish between the physical hypotheses of Sec. II and the rigorous mathematical theory of the present section, applicable to any functions and filters of the form discussed, since the majority of the rigorous results of this section apply not only to the longitudinal velocity but also to other variables.

We will also derive and discuss here a few results specific for the case when $u$ is the longitudinal velocity and $u_{\tau_{L}}$ is defined via a large-scale-filtered skin friction as in Eq. (2). We will use the terminology specific for this case so as to keep it consistent throughout the paper.

\section{A. Mean profile}

The mean velocity is defined as $U(y)=\langle u(t, x, y, z)\rangle$. From (5) one then gets $U(y)=$ $\left\langle u_{\tau_{L}} \tilde{u}\left(t u_{\tau_{L}}^{2}, x u_{\tau_{L}}, y u_{\tau_{L}}, z u_{\tau_{L}}\right)\right\rangle$. From now on the arguments of $u_{\tau_{L}}(t, x, z)$ [and of its fluctuation $\left.u_{\tau_{L}}^{\prime}(t, x, z)\right]$ will be omitted for brevity. Property (iv) allows us to insert the large-scale filter before averaging:

$$
U(y)=\left\langle\mathcal{L} u_{\tau_{L}} \tilde{u}\left(t u_{\tau_{L}}^{2}, x u_{\tau_{L}}, y u_{\tau_{L}}, z u_{\tau_{L}}\right)\right\rangle
$$

Property (v) then gives

$$
U(y)=\left\langle u_{\tau_{L}} \tilde{U}\left(y u_{\tau_{L}}\right)\right\rangle
$$

where $\tilde{U}(\tilde{y})$ is the average of $\tilde{u}(\tilde{t}, \tilde{x}, \tilde{y}, \tilde{z})$ over $\tilde{t}, \tilde{x}$, and $\tilde{z}$ with $\tilde{y}$ held constant: $\tilde{U}(\tilde{y})=\langle\tilde{u}(\tilde{t}, \tilde{x}, \tilde{y}, \tilde{z})\rangle$. Note again the use of mute variables in the definition of $\tilde{U}$ but not in Eq. (6), where $\tilde{U}$ has a specific argument.

Note the particular use of properties (iv) and (v) in calculating the average: It is done in two steps, first performing large-scale filtering and then replacing the large-scale filtering with averaging with $u_{\tau_{L}}$ kept constant. This technique is widely used in the derivations in the present paper.

Differentiating (6) with respect to $y$ at $y=0$ gives

$$
\frac{d U}{d y}(0)=\left\langle u_{\tau_{L}}^{2}\right\rangle \frac{d \tilde{U}}{d y}(0)
$$


Since $\tau^{*} / \rho^{*}=v^{*} d U^{*} / d y^{*}$, after simple substitutions (7) gives ${ }^{1}$ that $d \tilde{U} / d y(0)=1$. This is similar to the well-known identity $d U^{+} / d y^{+}(0)=1$.

Since the statistical properties of $\tilde{u}$ are independent of $\operatorname{Re}, \tilde{U}$ is also independent of Re, thus representing the mean universal velocity profile. Since the statistical properties of $u_{\tau_{L}}$ depend on $\mathrm{Re},(6)$ describes the dependence of the mean velocity on Re. If one assumes that in a certain range of $y$ the universal velocity profile has a logarithmic behavior $\tilde{U}(y)=\frac{1}{x} \ln y+B$, then the mean velocity profile $U(y)$ will also have a logarithmic behavior given by the formula

$$
U(y)=\frac{1}{\varkappa} \ln y+B+\frac{1}{\varkappa}\left\langle u_{\tau_{L}} \ln u_{\tau_{L}}\right\rangle
$$

[since $\left\langle u_{\tau_{L}}\right\rangle=1$ by (4)]. The logarithmic law is usually written in wall units, that is, nondimensional units based on the dynamic velocity $u_{\tau}^{*}=\sqrt{\left\langle\tau^{*}\right\rangle / \rho^{*}}$. The relationship between the wall units and the units (4) used in the present paper is

$$
u=u^{+} /\left\langle u_{\tau_{L}}^{+}\right\rangle, \quad t=t^{+}\left\langle u_{\tau_{L}}^{+}\right\rangle^{2}, \quad(x, y, z)=\left(x^{+}, y^{+}, z^{+}\right)\left\langle u_{\tau_{L}}^{+}\right\rangle .
$$

Note that

$$
\left\langle u_{\tau_{L}}^{+}\right\rangle=1 / \sqrt{\left\langle u_{\tau_{L}}^{2}\right\rangle}=1 / \sqrt{1+\left\langle u_{\tau_{L}}^{\prime 2}\right\rangle}
$$

where $u_{\tau_{L}}^{\prime}$ is the fluctuation with zero mean so that $u_{\tau_{L}}=1+u_{\tau_{L}}^{\prime}$. This follows from another useful relation $\left\langle u_{\tau_{L}}^{+2}\right\rangle=1$, which can be obtained from the definitions of $u_{\tau_{L}}^{*}$ and wall units. In wall units (8) becomes

$$
U^{+}\left(y^{+}\right)=\frac{\left\langle u_{\tau_{L}}^{+}\right\rangle}{\varkappa} \ln y^{+}+B+\frac{1}{\varkappa}\left\langle u_{\tau_{L}}^{+} \ln u_{\tau_{L}}^{+}\right\rangle .
$$

Assuming that $u_{\tau_{L}}^{\prime} \ll 1$ allows further simplification. More comments will be made in Sec. VI. Here we only conclude that the above analysis gives a rational justification for the dependence of the logarithmic-law constants on Re and allows us to relate them to the parameters of the large-scale fluctuations.

\section{B. Fluctuations}

By property (v), applying the large-scale filter to (5) gives

$$
u_{L}(t, x, y, z)=u_{\tau_{L}} \tilde{U}\left(y u_{\tau_{L}}\right)
$$

where $u_{L}(t, x, y, z)$ is the large-scale-filtered velocity. This includes the mean velocity, so the largescale fluctuation velocity is obtained by subtracting (6) from (11):

$$
u_{L}^{\prime}(t, x, y, z)=u_{L}(t, x, y, z)-U(y)=u_{\tau_{L}} \tilde{U}\left(y u_{\tau_{L}}\right)-\left\langle u_{\tau_{L}} \tilde{U}\left(y u_{\tau_{L}}\right)\right\rangle .
$$

The squared root mean square of the large-scale-filtered velocity is therefore

$$
u_{L, \mathrm{rms}}^{2}(y)=\left\langle u_{L}^{\prime 2}\right\rangle=\left\langle u_{\tau_{L}}^{2} \tilde{U}^{2}\left(y u_{\tau_{L}}\right)\right\rangle-\left\langle u_{\tau_{L}} \tilde{U}\left(y u_{\tau_{L}}\right)\right\rangle^{2}
$$

Subtracting (6) from (5) gives the total fluctuation velocity

$$
u^{\prime}=u-U=u_{\tau_{L}} \tilde{u}\left(t u_{\tau_{L}}^{2}, x u_{\tau_{L}}, y u_{\tau_{L}}, z u_{\tau_{L}}\right)-\left\langle u_{\tau_{L}} \tilde{U}\left(y u_{\tau_{L}}\right)\right\rangle
$$

and by definition the small-scale fluctuation velocity is $u_{S}^{\prime}=u^{\prime}-u_{L}^{\prime}$. Obviously, $\mathcal{L} u_{S}^{\prime}=0$; from this and property (v) it follows that

$$
\left\langle u_{S}^{\prime} u_{L}^{\prime}\right\rangle=0
$$

${ }^{1}$ This is one point where we do use the physical meaning of $u_{\tau_{L}}^{*}$ and $u^{*}$. If, for example, $u^{*}$ stood for the spanwise velocity component, then $\tilde{U}$ would be zero and as would its derivative. 
that is, small-scale and large-scale fluctuation velocities do not correlate. We define the universal fluctuation velocity as $\tilde{u}^{\prime}(\tilde{t}, \tilde{x}, \tilde{y}, \tilde{z})=\tilde{u}(\tilde{t}, \tilde{x}, \tilde{y}, \tilde{z})-\tilde{U}(\tilde{y})$ and define the square of the root-meansquare universal velocity fluctuation as $\tilde{u}_{\mathrm{rms}}^{2}(\tilde{y})=\left\langle\tilde{u}^{\prime 2}(\tilde{t}, \tilde{x}, \tilde{y}, \tilde{z})\right\rangle$, where the averaging is done over the statistical ensemble. The averaged value depends on $y$ but is independent of $\tilde{t}, \tilde{x}$, and $\tilde{z}$ as these directions (including time) are considered to be statistically homogeneous, so the ensemble averaging is equivalent to averaging over any one or any combination of these variables.

The small-scale velocity fluctuation can now be expressed as

$$
u_{S}^{\prime}=u_{\tau_{L}} \tilde{u}^{\prime}\left(t u_{\tau_{L}}^{2}, x u_{\tau_{L}}, y u_{\tau_{L}}, z u_{\tau_{L}}\right)
$$

The squared root mean square of the small-scale fluctuating velocity is therefore $u_{S, \text { rms }}^{2}(y)=$ $\left\langle u_{\tau_{L}}^{2} \tilde{u}_{\mathrm{rms}}^{2}\left(y u_{\tau_{L}}\right)\right\rangle$. Taking into account (13) gives that $u_{\mathrm{rms}}^{2}(y)=u_{S, \mathrm{rms}}^{2}(y)+u_{L, \mathrm{rms}}^{2}(y)$.

Equations (12) and (14) also allow two-point correlations to be expressed via the properties of the universal functions and $u_{\tau_{L}}$. For example,

$$
\left\langle u_{L}^{\prime}(t, x, y, z) u_{L}^{\prime}\left(t, x, y_{0}, z\right)\right\rangle=\left\langle u_{\tau_{L}}^{2} \tilde{U}\left(y u_{\tau_{L}}\right) \tilde{U}\left(y_{0} u_{\tau_{L}}\right)\right\rangle-\left\langle u_{\tau_{L}} \tilde{U}\left(y u_{\tau_{L}}\right)\right\rangle\left\langle u_{\tau_{L}} \tilde{U}\left(y_{0} u_{\tau_{L}}\right)\right\rangle
$$

and

$$
\left\langle u_{L}^{\prime}\left(t, x, y_{\mathrm{o}}, z\right){u_{S}^{\prime}}^{2}\left(t, x, y_{\mathrm{o}}, z\right)\right\rangle=\left\langle\left(u_{\tau_{L}} \tilde{U}\left(y_{\mathrm{o}}\right)-\left\langle u_{\tau_{L}} \tilde{U}\left(y_{\mathrm{o}}\right)\right\rangle\right) u_{\tau_{L}}^{2} \tilde{u}_{\mathrm{rms}}^{2}\left(y u_{\tau_{L}}\right)\right\rangle .
$$

In these cases the averaged characteristics of the actual velocity field can be expressed via the properties of $u_{\tau_{L}}$ and the averaged characteristics of the universal field $\tilde{u}$, while the full knowledge of $\tilde{u}$ is not required. However, knowledge of $\tilde{U}$ and $\tilde{u}_{\text {rms }}$ is not always enough: For example, the formula for two-point correlation of the small-scale velocity $\left\langle u_{S}^{\prime}(t, x, y, z) u_{S}^{\prime}\left(t, x, y_{0}, z\right)\right\rangle$ involves the two-point correlation of $\tilde{u}$. The above derivations illustrate the general approach, while the particular formulas will be used in the following sections.

\section{Expansion in the amplitude of large-scale fluctuations}

Equation (5), expressing the QSQH hypothesis, and formulas that can be derived from it for arbitrary $u_{\tau_{L}}$, for example, those in Secs. III A and III B, are neat and universal. However, they are difficult to interpret and use, since determining the statistical characteristics of $\tilde{u}(\tilde{t}, \tilde{x}, \tilde{y}, \tilde{z})$ is not easy. Fortunately, in many flows the amplitude of the fluctuation of the large-scale component of the skin friction is not large. This allows significant simplifications.

Since $\left\langle u_{\tau_{L}}\right\rangle=1$ by the nondimensionalization, the fluctuation $u_{\tau_{L}}^{\prime}=u_{\tau_{L}}-1$. Assuming $u_{\tau_{L}}^{\prime} \ll 1$, one can use asymptotic expansions. Thus, (6) reduces to

$$
U(y)=\tilde{U}(y)+\left\langle u_{\tau_{L}}^{\prime 2}\right\rangle \frac{d}{d y}\left(\frac{y^{2}}{2} \frac{d \tilde{U}}{d y}\right)+\cdots
$$

and (11) reduces to

$$
u_{L}^{\prime}=u_{\tau_{L}}^{\prime}\left(\tilde{U}(y)+y \frac{d \tilde{U}}{d y}\right)+\left(u_{\tau_{L}}^{\prime 2}-\left\langle u_{\tau_{L}}^{\prime 2}\right\rangle\right) \frac{d}{d y}\left(\frac{y^{2}}{2} \frac{d \tilde{U}}{d y}\right)+\cdots .
$$

The square of the root-mean-square velocity fluctuation can be expressed as

$$
u_{\mathrm{rms}}^{2}(y)=\tilde{u}_{\mathrm{rms}}^{2}(y)+\left\langle u_{\tau_{L}}^{\prime 2}\right\rangle\left[\left(\tilde{U}(y)+y \frac{d \tilde{U}}{d y}\right)^{2}+\frac{1}{2} \frac{d^{2}\left(y^{2} \tilde{u}_{\mathrm{rms}}^{2}\right)}{d y^{2}}\right]+\cdots .
$$

Chernyshenko et al. [27] neglected all but the main terms. This leads to $\tilde{U}(y) \approx U(y)$ and $\tilde{u}_{\mathrm{rms}}^{2}(y) \approx u_{\mathrm{rms}}^{2}(y)$, thus allowing $\tilde{U}(y)$ and $\tilde{u}_{\mathrm{rms}}^{2}(y)$ to be found. In fact, we will see that at least for the set of data and the filter we will use only the first of these approximate equalities is satisfied with good accuracy, while the second is not: The first term in the square brackets of (17) cannot be neglected. We will also see that neglecting the second-order terms in Eq. (16) is justified. 
This leads to

$$
u_{L}^{\prime} \approx u_{\tau_{L}}^{\prime}\left(\tilde{U}(y)+y \frac{d \tilde{U}}{d y}\right) .
$$

These observations will affect our choice of the filter.

A similar expansion for the small-scale fluctuation (14) leads to

$$
u_{S}^{\prime}=\left(1+u_{\tau_{L}}^{\prime}\right)\left[\tilde{u}^{\prime}(t, x, y, z)+u_{\tau_{L}}^{\prime}\left(2 t \frac{\partial \tilde{u}^{\prime}}{\partial t}+x \frac{\partial \tilde{u}^{\prime}}{\partial x}+y \frac{\partial \tilde{u}^{\prime}}{\partial y}+z \frac{\partial \tilde{u}^{\prime}}{\partial z}\right)+\cdots\right]
$$

and we face the difficulty of nonconvergence at large values of $t$ or spatial coordinates. For homogeneous directions and time this could be circumvented by selecting the origin of $t, x$, and $z$ to be at the point of interest, at least as far as the multipoint statistics of interest have only $y$ different at these points, as it is the case in the present paper. The problem with the wall-normal direction is more serious. Our comparisons will often be done up to and somewhat beyond $y=100$. This is not a problem for large-scale or averaged quantities because, for example, the term $y d \tilde{U} / d y$ is not large for large $y$ since $d \tilde{U} / d y$ is small where $y$ is large. However, $\partial \tilde{u}^{\prime} / \partial y$ might not be small at high $y$ and this might be even more true for the higher derivatives. For this reason, for statistics involving the small-scale component it is safer first to obtain the general formula for it and only then perform the expansion, as done, for example, in deriving (17). Hence, in this paper we will use the full expression (14) for the small-scale fluctuations even when an expansion in small $u_{\tau_{L}}^{\prime}$ is considered.

\section{Comparison with empirical relationships}

Neglecting the higher-order terms, the QSQH hypothesis can be expressed [27] in the form very similar to the empirical formula (1). For this, (18) should be used at the location $y=y_{0}$ of the outer probe to express $u_{\tau_{L}}^{\prime}$ via $u_{L}^{\prime+}\left(y_{\mathrm{o}}^{+}\right): u_{\tau_{L}}^{\prime}=u_{L}^{\prime+}\left(y_{\mathrm{o}}^{+}\right) /\left[\tilde{U}\left(y_{\mathrm{o}}\right)+y_{\mathrm{o}} d \tilde{U} / d y_{\mathrm{o}}\right]$. Then a variable $u_{\mathrm{u}, \mathrm{QS}}^{\prime}$ is introduced satisfying the equation

$$
\tilde{u}\left(t u_{\tau_{L}}^{2}, x u_{\tau_{L}}, y u_{\tau_{L}}, z u_{\tau_{L}}\right)=\frac{\tilde{u}_{\mathrm{rms}}\left(y u_{\tau_{L}}\right)}{\tilde{u}_{\mathrm{rms}}(y)} u_{\mathrm{u}, \mathrm{QS}}^{\prime} .
$$

The idea is that the amplitude of the fluctuations of $u_{\mathrm{u}, \mathrm{QS}}^{\prime}$ will be unaffected by $u_{\tau_{L}}^{\prime}$, which is the expected property of $u_{\mathrm{u}}^{\prime}$ in Eq. (1). These equations are then substituted into the expressions (14) and (18) for small- and large-scale fluctuations, which then are added together, $\tilde{u}_{\mathrm{rms}}\left(y u_{\tau_{L}}\right)$ is expanded in $u_{\tau_{L}}^{\prime}$, and only the linear terms are kept. From (9) and (10) it follows that up to the higher-order terms the units (4) used in the present paper and the wall units coincide. This leads to the expression for the velocity fluctuation coinciding with Eq. (1), provided $u_{\mathrm{u}, \mathrm{QS}}^{\prime}=u_{\mathrm{u}}^{\prime}$,

$$
\alpha(y)=\frac{\tilde{U}(y)+y \frac{d \tilde{U}}{d y}}{\tilde{U}\left(y_{\mathrm{o}}\right)+y_{\mathrm{o}} \frac{d \tilde{U}}{d y_{\mathrm{o}}}}, \quad \beta(y)=\frac{1+\frac{y}{\tilde{u}_{\mathrm{rms}}(y)} \frac{d \tilde{u}_{\mathrm{rms}}(y)}{d y}}{\tilde{U}\left(y_{\mathrm{o}}\right)+y_{\mathrm{o}} \frac{d \tilde{U}}{d y_{\mathrm{o}}}} .
$$

Assuming that $u_{\mathrm{u}, \mathrm{QS}}^{\prime}=u_{\mathrm{u}}^{\prime}$ is not rigorous. Chernyshenko et al. [27] commented that $u_{\mathrm{u}}^{\prime}$ in Eq. (1) is Re independent, while $u_{\mathrm{u}, \mathrm{QS}}^{\prime}$ is frequency and scale modulated. Frequency modulation was also considered by Ganapathisubramani et al. [24]. Another subtle difference lies in the definition of the amplitude of the fluctuations, which is usually defined via the Hilbert transform in the context of (1) and as $u_{\tau_{L}} \tilde{u}_{\text {rms }}\left(y u_{\tau_{L}}\right)$ in Eq. (19); these definitions, while similar, are not exactly the same. The relationships between various indicators of the amplitude of fluctuation in the present context were discussed in a number of works [29-32]. In spite of these subtleties, it is clear that the linearized QSQH hypothesis does provide a theoretical justification for the empirical model (1). A more complicated empirical model proposed in Ref. [26] is nonlinear and if it can also be explained by the QSQH hypothesis, this will be more complicated. 

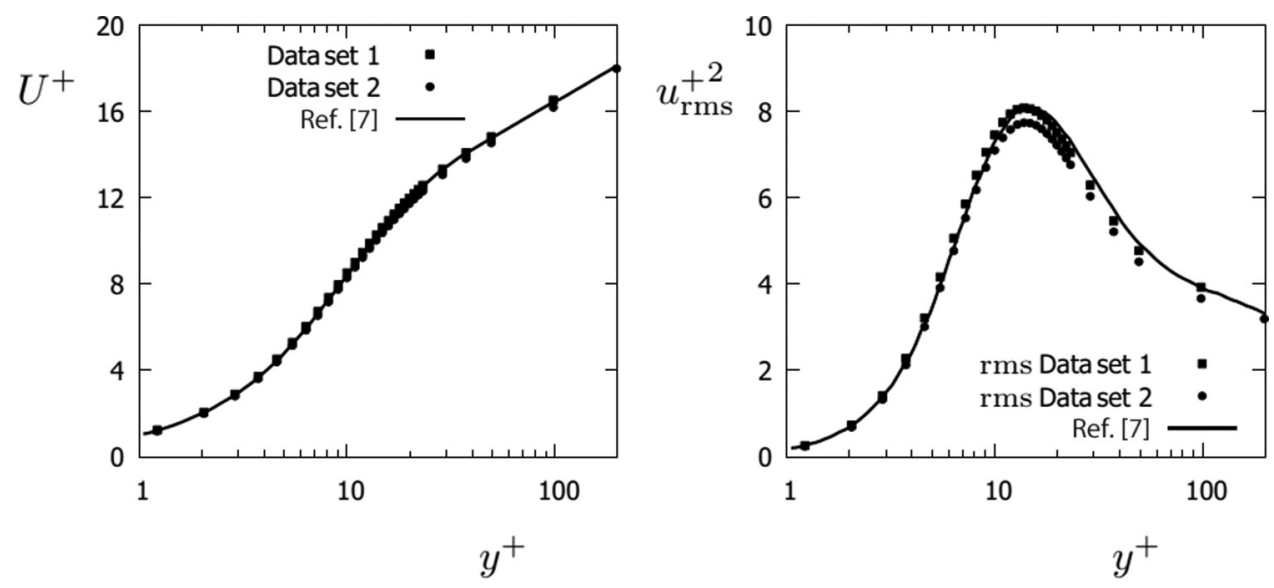

FIG. 2. Mean velocity $U^{+}$and root-mean-square fluctuations $u_{\mathrm{rms}}^{+2}$ as a function of wall distance. The points are obtained from our data set and the curves are from [7]. The discrepancy between the curves characterizes the accuracy of the data we used.

\section{DATA SET AND SELECTION OF THE LARGE-SCALE FILTER}

The motivation of investigating the possibility to use the QSQH hypothesis for developing means of extrapolating the results of direct numerical simulations of flows with drag reduction to higher Re led us to choosing the set of data containing the drag-reduction case, described in detail in Ref. [33] and provided to us by the authors of that paper. In the present work, however, we use only the part of database describing the reference flow with no drag reduction, which is the canonical channel flow at $\operatorname{Re}_{\tau}=1015$ in a computational domain of the (length) $\times$ (height) $\times($ width $)=4 \pi h \times 2 h \times 2 \pi h$, with a duration of about $T^{+}=2600$. This duration is somewhat short for certain tasks: Therefore, we use two approximately equal halves of it separately and compare the results to verify that the averaging error is sufficiently small so as not to affect the conclusions of this paper. Figure 2 gives a comparison of the mean velocity profile and the root-mean-square fluctuation obtained for the two parts of our data set with the data available in the literature. The discrepancy gives an idea about the magnitude of error of our data set.

\section{A. Applying the filter of Marusic and co-workers to the data set}

The spectral filter suggested by Hutchins and Marusic [21] cuts off the Fourier components with the streamwise wavelength $\lambda_{x}^{+}$smaller than the channel half-width. The cutoff length is thus between the wavelength of inner and outer spectral peaks, which correspond to the dominant small and large scales of near-wall turbulence. Also, a Fourier filter has the required properties (i)-(iv) of an ideal filter. Hutchins and Marusic suggested using this filter at $\operatorname{Re}_{\tau}>1700$ because the largeand small-scale components are not fully separated in low- $\mathrm{Re}_{\tau}$ flows. Comparing the predictions of the QSQH theory with empirical data for $\alpha\left(y^{+}\right)$and $\beta\left(y^{+}\right)$in Eq. (1), which were obtained by using this filter, gave a reasonable, but not perfect, agreement [27]. We performed the same comparisons for our data set, but the agreement with the QSQH theory was not as good. To further examine the properties of this filter we selected two wall-parallel layers. The first layer was very close to the wall (at the nearest grid layer, denoted below by $y^{+}=0$ ) and the second layer was at $y^{+}=100$. Figure 3 shows the instantaneous large-scale fluctuation of $u$ given by this filter at $y^{+}=0$ and $y^{+}=100$. According to (18), the large-scale-filtered velocity at different $y$ should differ by a factor independent of $t, x$, and $z$. This is clearly not the case in Fig. 3. The details of these pictures reveal the reason for this: The cutoff length is not large enough compared to the length of near-wall streaks and hence it does not remove them. For higher values of $\operatorname{Re}_{\tau}$ such a filter might work, but to apply 

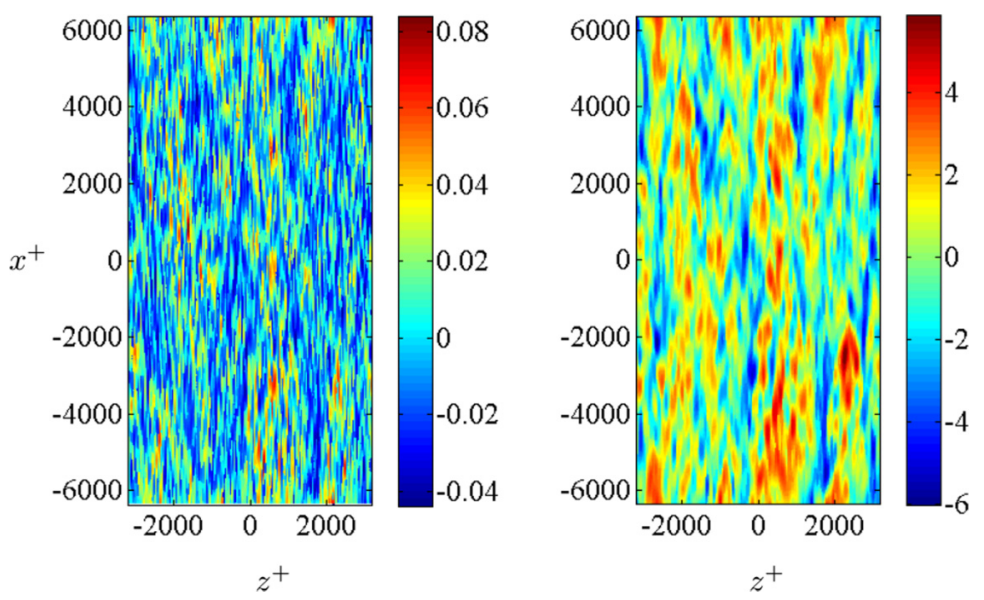

FIG. 3. Large-scale velocity fluctuations obtained by applying the Hutchins-Marusic [21] filter at the layers of $y^{+}=0$ (left) and $y^{+}=100$ (right).

the QSQH theory for the case in question, a different filter is needed. Fortunately, since we have access to a database of a direct numerical simulation containing the entire velocity field, this turns out to be possible.

\section{B. Optimal filter}

The available numerical data allows cutoff Fourier filtering in wall-parallel directions as well as cutoff Fourier filtering in time. This requires defining three thresholds. For any cutoff thresholds a Fourier filter satisfies the first four requirements stated in Sec. II B. Property (v) can be satisfied only approximately. As a quantitative measure of how well it is satisfied we selected the correlation coefficient $r_{L L}=\left\langle\left.\left. u_{L}^{\prime}\right|_{y^{+}=0^{+}} u_{L}^{\prime}\right|_{y^{+}=y_{0}^{+}}\right\rangle / \sqrt{\left\langle u_{L}^{\prime 2}\right\rangle\left\langle u_{L}^{\prime 2}\right\rangle}$ between the large-scale field very close to the wall and the large-scale field at the outer layer at $y^{+}=y_{\mathrm{o}}^{+}=100$, since we know from Sec. IV A that it is a sensitive measure of the filter quality. From (18) it follows that this correlation coefficient should be close to 1 , provided property (v) is satisfied and $\left\langle u_{\tau_{L}}^{\prime 2}\right\rangle$ is small enough. We observed that by increasing the threshold levels it is possible to make the correlation coefficient very close to 1 , however, increasing the thresholds decreases the energy of the large-scale fluctuations, thus making the results less physically significant. Another obvious effect of increasing the thresholds is an increase of the correlation between the small-scale component at the wall and the small-scale component at the outer layer, $r_{S S}=\left\langle\left.\left. u_{L}^{\prime}\right|_{y^{+}=0^{+}} u_{S}^{\prime}\right|_{y^{+}=y_{0}^{+}}\right\rangle / \sqrt{\left\langle u_{S}^{\prime 2}\right\rangle\left\langle u_{S}^{\prime 2}\right\rangle}$. The absence of correlation between the small-scale motions at the points separated by large distance is naturally expected. Hence, we sought a compromise between $r_{L L}$ being as close to 1 as possible and $r_{S S}$ being small.

Calculations for different cutoff thresholds were performed. The large-scale to large-scale and small-scale to small-scale correlations of each cutoff point were plotted and the Pareto front based on these points is shown in Fig. 4.

The Pareto front is a standard tool for multiobjective optimisation. The axes are the values of the objective functions that are desired to be minimized or maximized. In our case the abscissa is $r_{L L}$ and the ordinate is $r_{S S}$. Each filter gives a certain combination of $r_{L L}$ and $r_{S S}$, that is, a point in the $r_{L L}-r_{S S}$ plane. The Pareto front is the boundary between those points $\left(r_{L L}, r_{S S}\right)$ that can be obtained with at least one filter and those points $\left(r_{L L}, r_{S S}\right)$ that cannot be obtained with any filter. In our case, we would like to have the point $\left(r_{L L}, r_{S S}\right)$ be as far to the right as possible and as far down as possible. Any point above and to the left of the Pareto front is not optimal, since both $r_{L L}$ and $r_{S S}$ can be improved by selecting a different filter. Points on the Pareto front, however, correspond to filters such that none of the objective function values can be improved without worsening the other. 


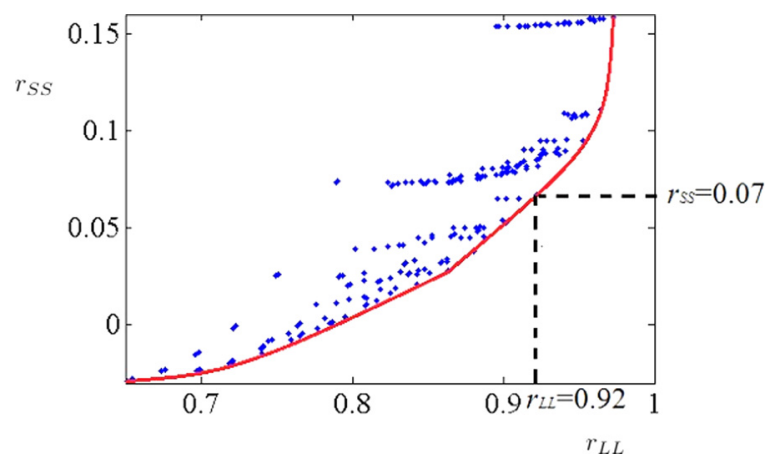

FIG. 4. Pareto front of large-large and small-small correlation coefficients, where each point represents a combination of cutoff frequency and wave numbers.

Hence, the optimal point has to be on the Pareto front, but the further choice has to be done on the basis of additional criteria.

Along the Pareto front, the values of large-scale to large-scale correlation vary between 0.65 and 0.97 and those of small-scale to small-scale correlation vary between -0.03 and 0.15 . We selected the point shown in the figure as a reasonable compromise. At this point the frequency and wave-number cutoff in Fourier-transform space correspond to the time scale $T_{c}^{+}=260$, the longitudinal length $X_{c}^{+}=1000 \pi$, and the spanwise length $Z_{c}^{+}=125 \pi$.

Applying the new filter leads to a noticeable improvement, as shown in Fig. 5. The thin streaks that appeared in Fig. 3 are not observed anymore. The size, shape, and position of the large-scale structures at the near-wall layer and the outer layer are close.

For our filter, $\left\langle u_{\tau_{L}}^{\prime 2}\right\rangle=0.004364$, which means that (18) is a good approximation of (16). From (18) it follows that $r_{u_{L} u_{\tau_{L}}}(y)=\left\langle u_{L}^{\prime} u_{\tau_{L}}^{\prime}\right\rangle / \sqrt{\left\langle u_{L}^{\prime 2}\right\rangle\left\langle u_{\tau_{L}}^{\prime}\right\rangle^{2}} \approx 1$. Figure 6 shows that the discrepancy is within $10 \%$. This level of accuracy is acceptable for studying physical mechanisms of the flow. However, it should be admitted that the choice of the current large-scale filter is still somewhat arbitrary. Therefore, we will also examine the influence of the filter on the accuracy of QSQH predictions in the next section, where we will also show results for another filter corresponding to the point on the Pareto front at $r_{L L}=0.78$.
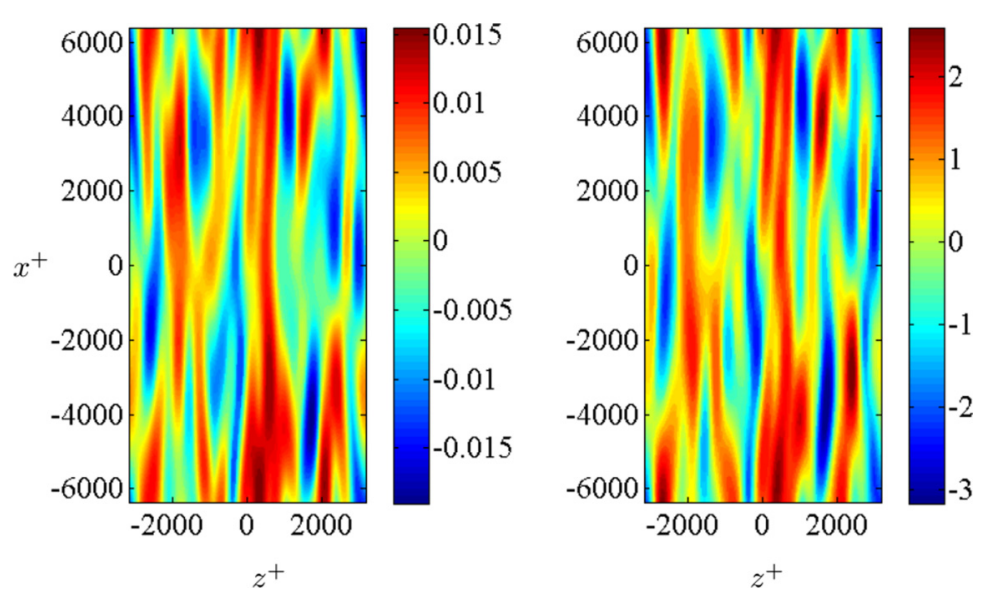

FIG. 5. Large-scale velocity fluctuations obtained by the new filter at the layers of $y^{+}=0$ (left) and $y^{+}=100$ (right). 


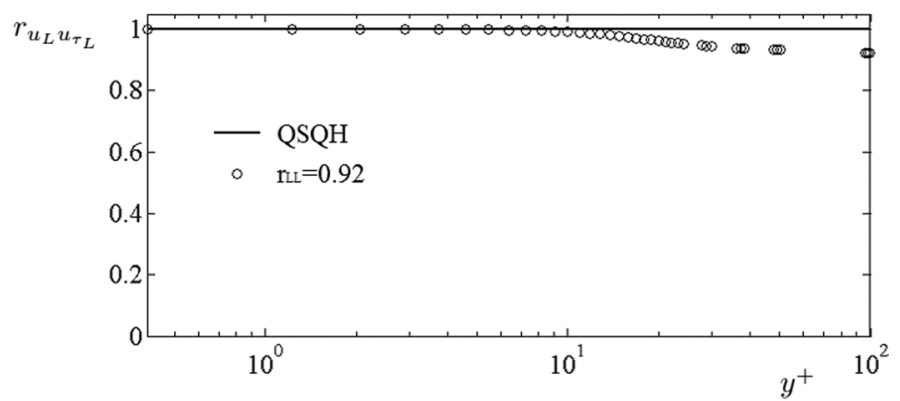

FIG. 6. Comparison for $r_{u_{L} u_{\tau_{L}}}$ as given by the theory (line) and the numerical data of $r_{L L}=0.92$ (circles).

\section{COMPARISONS}

The nondimensional units (4), convenient for the QSQH theory, differ from the wall units [see (9) and (10)] by a factor $\sqrt{1+\left\langle u_{\tau_{L}}^{\prime 2}\right\rangle} \approx 1.002$ for the case in question. Such a difference is not visible on the plots and will be ignored: The plot axes will refer to the familiar wall units.

\section{A. Mean universal velocity profile and mean universal root-mean-square fluctuation}

The universal mean velocity profile $\tilde{U}(y)$ can be found from (15), in which the term quadratic in $u_{\tau_{L}}^{\prime}$ can be neglected. To estimate this quadratic term, we can assume that $\tilde{U}(y) \approx \frac{1}{x} \ln y+B$ away from the wall. Then, for $\varkappa=0.4$, this term equals $\left\langle u_{\tau_{L}}^{\prime 2}\right\rangle / 2 \varkappa \approx 0.0055$, which is much smaller than $U(y)$. One can use (7) to demonstrate that the quadratic term is also negligible near the wall. Hence, with good accuracy we will take $\tilde{U}(y)=U(y)$. This also justifies the use of the linearized expression (18) for the large-scale fluctuation.

The situation changes for $\tilde{u}_{\text {rms }}$. In this case [see (17)], the quadratic term involving $\tilde{u}_{\text {rms }}$ can indeed be neglected, but $\left\langle u_{\tau_{L}}^{\prime 2}\right\rangle[\tilde{U}(y)+y d \tilde{U} / d y]^{2}$ is relatively large. At $y=100$, taking $B=5$, the logarithmic law gives a value of about 1.6 for this term, while the maximum value of $u_{\mathrm{rms}}^{2}(y)$ is below 10 for $\operatorname{Re}_{\tau}=1020$ of our data set. Hence, we take

$$
\tilde{u}_{\mathrm{rms}}^{2}(y) \approx u_{\mathrm{rms}}^{2}(y)-\left\langle u_{\tau_{L}}^{\prime 2}\right\rangle\left(\tilde{U}(y)+y \frac{d \tilde{U}}{d y}\right)^{2} .
$$

The large factor $[\tilde{U}(y)+y d \tilde{U} / d y]^{2}$ will also enter other formulas, where also it cannot be neglected. As we will see, this makes a significant difference.

Figure 7 shows $\tilde{u}_{\text {rms }}^{+2}$ given by (21) for the two parts of our data set; $\tilde{u}_{\mathrm{rms}}^{+2}$ turns out to be quite close to the actual root-mean-square fluctuations at $\operatorname{Re}_{\tau}=182$ [7], also shown in the figure. One can argue that this could be expected because at such a low Re there are no large-scale fluctuations.

\section{B. Large-scale motion}

The first and most obvious target for comparisons is the coefficient $\alpha(y)$ in Eq. (1) introduced by Hutchins and Marusic [21]:

$$
\alpha(y)=\frac{\left\langle u_{L}^{\prime}(y) u_{L}^{\prime}\left(y_{\mathrm{o}}\right)\right\rangle}{\left\langle u_{L}^{\prime 2}\left(y_{\mathrm{o}}^{2}\right)\right\rangle}
$$

(we ignore here the small difference between $y$ and $y^{+}$). This coefficient is the two-point correlation of the large-scale velocity normalized in a certain way, with $y$ and $y_{\mathrm{o}}$ usually interpreted as the position of the wall probe and the position of the outer probe, respectively. The agreement with linearized QSQH theory prediction, $\alpha(y) \approx[\tilde{U}(y)+y d \tilde{U} / d y] /\left[\tilde{U}\left(y_{\mathrm{o}}\right)+y_{\mathrm{o}} d \tilde{U} / d y_{\mathrm{o}}\right]$, as shown in Fig. 1, is rather good. The second-order term in the expression for $\alpha(y)$ is negligible. However, we 

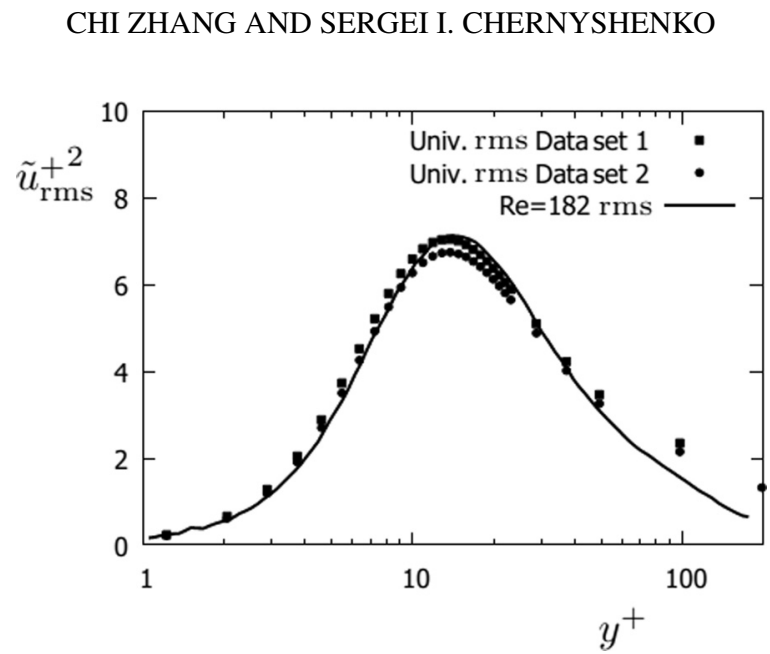

FIG. 7. Universal rms fluctuations $\tilde{u}_{\mathrm{rms}}^{+2}$ by (21) for two data subsets (points) and actual rms fluctuations $u_{\mathrm{rms}}^{+2}$ for $\operatorname{Re}_{\tau}=182$ from [7] (curve).

have already mentioned that the good agreement in Fig. 1 is in part coincidental. The coefficient $\alpha(y)$ defined by (22) is such that $\alpha(0) \equiv 0$ and $\alpha\left(y_{\mathrm{o}}\right) \equiv 1$. Accordingly, any reasonable theory will have zero errors at these two points and this reduces also the error in between. Several other nondimensional coefficients can also characterize the behavior of the large-scale fluctuations. We introduce

$$
\begin{aligned}
& \alpha_{0}(y)=\frac{\left\langle u_{L}^{\prime}(y) u_{L}^{\prime}\left(y_{\mathrm{o}}\right)\right\rangle}{\left\langle u_{L}^{\prime 2}\left(y_{\mathrm{o}}^{2}\right)\right\rangle}\left(\tilde{U}\left(y_{\mathrm{o}}\right)+y_{\mathrm{o}} \frac{d \tilde{U}}{d y_{\mathrm{o}}}\right) \approx \alpha(y)\left(\tilde{U}\left(y_{\mathrm{o}}\right)+y_{\mathrm{o}} \frac{d \tilde{U}}{d y_{\mathrm{o}}}\right), \quad \alpha_{1}(y)=\frac{\left\langle u_{\tau_{L}}^{\prime} u_{L}^{\prime}(y)\right\rangle}{\left\langle u_{\tau_{L}}^{\prime 2}\right\rangle}, \\
& \alpha_{2}(y)=\sqrt{\frac{\left\langle u_{L}^{\prime 2}(y)\right\rangle}{\left\langle u_{\tau_{L}}^{\prime 2}\right\rangle}}, \quad \alpha_{3}(y)=\sqrt{\frac{\left\langle u_{L}^{\prime 2}(y)\right\rangle}{\left\langle u_{L}^{\prime 2}\left(y_{\mathrm{o}}\right)\right\rangle}}\left(\tilde{U}\left(y_{\mathrm{o}}\right)+y_{\mathrm{o}} \frac{d \tilde{U}}{d y_{\mathrm{o}}}\right) .
\end{aligned}
$$

For these functions the higher-order terms of the expansion in $u_{\tau_{L}}^{\prime}$ are negligible and the linearized QSQH theory gives the same expression:

$$
\alpha_{0}(y) \approx \alpha_{1}(y) \approx \alpha_{2}(y) \approx \alpha_{3}(y) \approx \tilde{U}(y)+y \frac{d \tilde{U}}{d y} .
$$

Figure 8 shows the comparison. Figures 6 and 8 give a more fair illustration of the accuracy of the QSQH hypothesis for the filter we use than Fig. 1. The error is about $10 \%$ under $y^{+}=100$ and in fact for Fig. 6 it is the value we ourselves selected by choosing a point on the Pareto front in Fig. 4 such that $r_{L L}$ was 0.92 . What should be mentioned is that adding the second-order term into

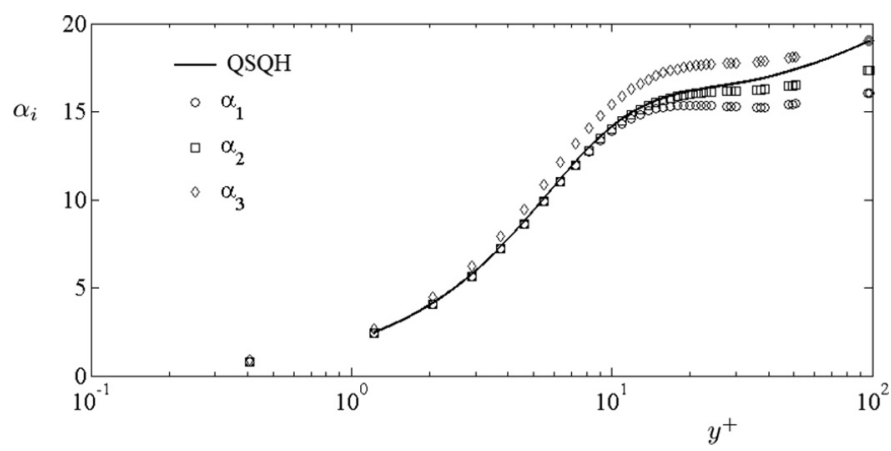

FIG. 8. Comparison: The solid curve is the linearized QSQH theory prediction (23) and points are $\alpha_{1}$ (circles), $\alpha_{2}$ (squares), and $\alpha_{3}$ (diamonds). The quality of the agreement for $\alpha_{0}$ can be inferred from Fig. 1. 


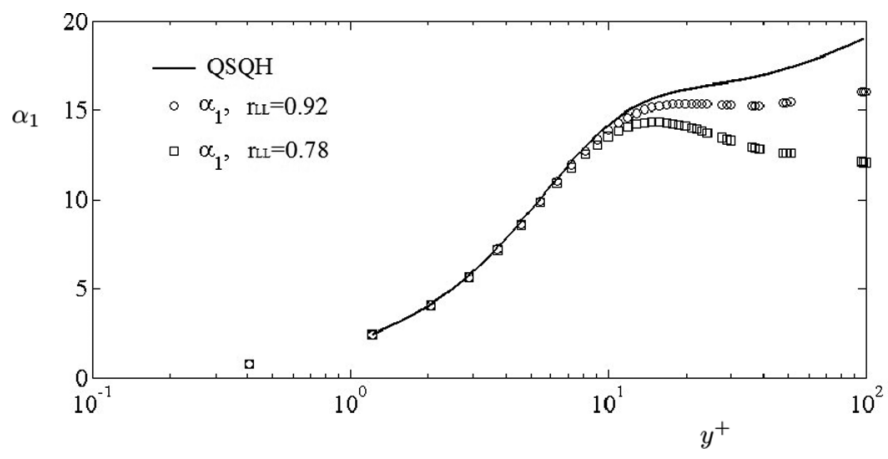

FIG. 9. Comparison: The solid curve is the linearized QSQH theory prediction (23) and circles and squares are the $\alpha_{1}$ calculated with the large-scale filters of $r_{L L}=0.92$ and $r_{L L}=0.78$.

calculations has no noticeable effect on Fig. 8. However, if we choose a point with lower $r_{L L}$ on the left side of the Pareto front, the agreement between the $\alpha_{1}$ function and the QSQH prediction will deteriorate, as shown in Fig. 9. Here the $r_{L L}$ and $r_{S S}$ of the second point are 0.78 and 0 . Therefore, we can conclude that, in the framework of QSQH theory, there is a trade-off between the accuracy of the QSQH theory and the physical significance of the large-scale components. This trade-off is controlled by the position of the filter threshold point on the Pareto front.

\section{Modulation of fluctuation amplitude by large scales}

Various measures of the modulation of small-scale fluctuations by large scales can be used and were discussed [29-32,34]. We calculated the correlations between $u_{\tau_{L}}^{\prime}$ and the squares of small-scale fluctuation and the total fluctuations, normalized ${ }^{2}$ with $\left\langle u_{\tau_{L}}^{\prime 2}\right\rangle$ :

$$
\gamma_{1}(y)=\frac{\left\langle u_{\tau_{L}}^{\prime} u_{S}^{\prime 2}\right\rangle}{\left\langle u_{\tau_{L}}^{\prime 2}\right\rangle}, \quad \gamma_{2}(y)=\frac{\left\langle u_{\tau_{L}}^{\prime}\left(u^{\prime 2}-\overline{u^{\prime 2}}\right)_{L}\right\rangle}{\left\langle u_{\tau_{L}}^{\prime 2}\right\rangle},
$$

where, as everywhere, the subscript $L$ denotes a large-scale-filtered component. Using the same derivation techniques as in the previous sections gives

$$
\begin{gathered}
\gamma_{1}(y)=2 \tilde{u}_{\mathrm{rms}}^{2}+y \frac{d \tilde{u}_{\mathrm{rms}}^{2}}{d y}+\frac{1}{2} \frac{\left\langle u_{\tau_{L}}^{\prime 3}\right\rangle}{\left\langle u_{\tau_{L}}^{\prime 2}\right\rangle} \frac{d^{2}\left(y^{2} \tilde{u}_{\mathrm{rms}}^{2}\right)}{d y^{2}}+\cdots, \\
\gamma_{2}(y)=\gamma_{1}(y)+\frac{\left\langle u_{\tau_{L}}^{\prime 3}\right\rangle}{\left\langle u_{\tau_{L}}^{\prime 2}\right\rangle}\left(\tilde{U}+y \frac{d \tilde{U}}{d y}\right)^{2}+\cdots .
\end{gathered}
$$

Note that the difference between $\gamma_{1}$ and $\gamma_{2}$ is proportional to the normalized skewness of $u_{\tau_{L}}^{\prime}$, which can be regarded as a first-order nonlinear term. The relation of the skewness and the measures of the amplitude modulation of the small scales is well known [29-31]. The last term in Eq. (25) is the same as the last term in the square brackets in Eq. (17). For the data set and the filter we are using this term turns out to be negligible. We can now use (21) to eliminate $\tilde{u}_{\text {rms }}$ from (25) (with the second term in the square brackets neglected), arriving at

$$
\gamma_{1}(y) \approx \frac{1}{y} \frac{d}{d y}\left\{y^{2}\left[u_{\mathrm{rms}}^{2}-\left\langle u_{\tau_{L}}^{\prime 2}\right\rangle\left(\tilde{U}+y \frac{d \tilde{U}}{d y}\right)^{2}\right]\right\} .
$$

\footnotetext{
${ }^{2}$ Recall that the velocity is nondimensionalized with $\left\langle u_{\tau_{L}}\right\rangle$, so $\gamma_{1}$ can also be written in the form $\gamma_{1}=$ $\left\langle u_{\tau_{L}}^{\prime} u_{S}^{\prime 2}\right\rangle /\left(\left\langle u_{\tau_{L}}^{\prime 2}\right\rangle\left\langle u_{\tau_{L}}\right\rangle\right)$, independent of the units of measure.
} 
Here the last term inside the square brackets is a second-order nonlinear term. If only the main term of the expansion is kept, (26) and (27) reduce to

$$
\gamma_{1}(y) \approx \gamma_{2}(y) \approx \gamma(y)=\frac{1}{y} \frac{d}{d y}\left(y^{2} u_{\mathrm{rms}}^{2}\right) .
$$

A comparison with (20) shows that

$$
\gamma(y)=2 \beta(y) u_{\mathrm{rms}}\left(\tilde{U}\left(y_{\mathrm{o}}\right)+y_{\mathrm{o}} \frac{d \tilde{U}}{d y_{\mathrm{o}}}\right) .
$$

Hence, a separate comparison for $\beta(y)$ is not necessary.

Figure 10 shows the comparisons. For two large-scale filters with different values of $r_{L L}$, the behavior of $\gamma$ differs from the behavior of $\alpha$ in two respects. The first respect is that taking into account the higher-order terms leads to a substantial change of the results. Moreover, we found that the accuracy of QSQH predictions of $\gamma_{2}$ is better than that of $\gamma_{1}$. The difference between the linear and nonlinear curves of $\gamma_{1}$ (containing first-order correction only) and $\gamma_{2}$ (containing both firstand second-order corrections) shows that the contribution of the second-order nonlinear term, while smaller, remains comparable to the contribution of the first-order nonlinear term. This indicates that taking into account more higher-order terms might improve the agreement. The second respect is that choosing a different large-scale filter will affect not only the actual value of $\gamma$ obtained from DNS using (24), but also the QSQH predictions given by (25) and (26). It is interesting to note that in Eq. (27) the only term depending on the filter is $\left\langle u_{\tau_{L}}^{\prime 2}\right\rangle$ and, as it is obvious from the shape of the curves, adjusting the filter might result in a close agreement for $\gamma_{1}$. This, however, will also affect $\gamma_{2}$. Just as the figure shows, when the large-scale filter with different $r_{L L}$ was applied, the prediction error for $\gamma_{1}$ decreased and that for $\gamma_{2}$ increased. Overall, the error of the QSQH predictions for $\gamma$ functions is somewhat greater than the error for $\alpha$ functions, but unlike the $\alpha$ functions, it can be expected to be improved somewhat by taking the nonlinearity into account fully.

\section{Correlation reversal}

Intuitively, when the large-scale velocity increases the fluctuation intensity should increase too. However, the modulation coefficient $\beta(y)$ found by fitting experimental data [23] is positive near the wall but becomes negative farther away from it. This counterintuitive reversal of the correlation between large-scale fluctuations and the intensity of small-scale fluctuations is now well ascertained by experiments and direct numerical simulations. The QSQH hypothesis suggests [27] the physical mechanism of the correlation reversal illustrated in Fig. 11. According to (14), the effect of the change in the large-scale fluctuation is twofold: an amplitude modulation and a frequency or scale modulation. When $u_{\tau_{L}}$ increases, the maximum of the fluctuation intensity curve also increases due to amplitude modulation, but it also shifts towards the wall due to the modulation of the wall-normal length scale. Near the wall ( $y=a$ in Fig. 11) both effects increase the fluctuation intensity at a fixed distance to the wall, but farther away where the intensity decreases with wall distance $(y=b$ in Fig. 11), the scale-modulation effect works towards decreasing the fluctuation intensity. A somewhat similar mechanism was proposed in Ref. [6] for the reversal of the correlation of the intensity of the wall-normal fluctuations, where the effect of scale modulation was suggested to be leading to correlation reversal via the proxy of the shear, with which the fluctuation intensity was assumed to be in equilibrium. The expression for $\beta(y)$ [or $\gamma(y)$; see (29)] derived from the quasisteady hypothesis using linearization does describe a decrease in $\beta(y)$ as $y$ increases beyond a certain value, so the general shape of the curve is similar, but the predicted $\beta(y)$ remains positive everywhere. When the nonlinear terms of the expansion are taken into account, the situation becomes more complicated, because various modulation measures might become or not become negative at a certain distance from the wall (see Fig. 10), but in any case the nonlinearity improves the agreement. On the other hand, there are alternative explanations of the correlation reversal, related to the phase shift between the large and small scales $[24,32,35]$. Also, there is a possible discrepancy between the implications 

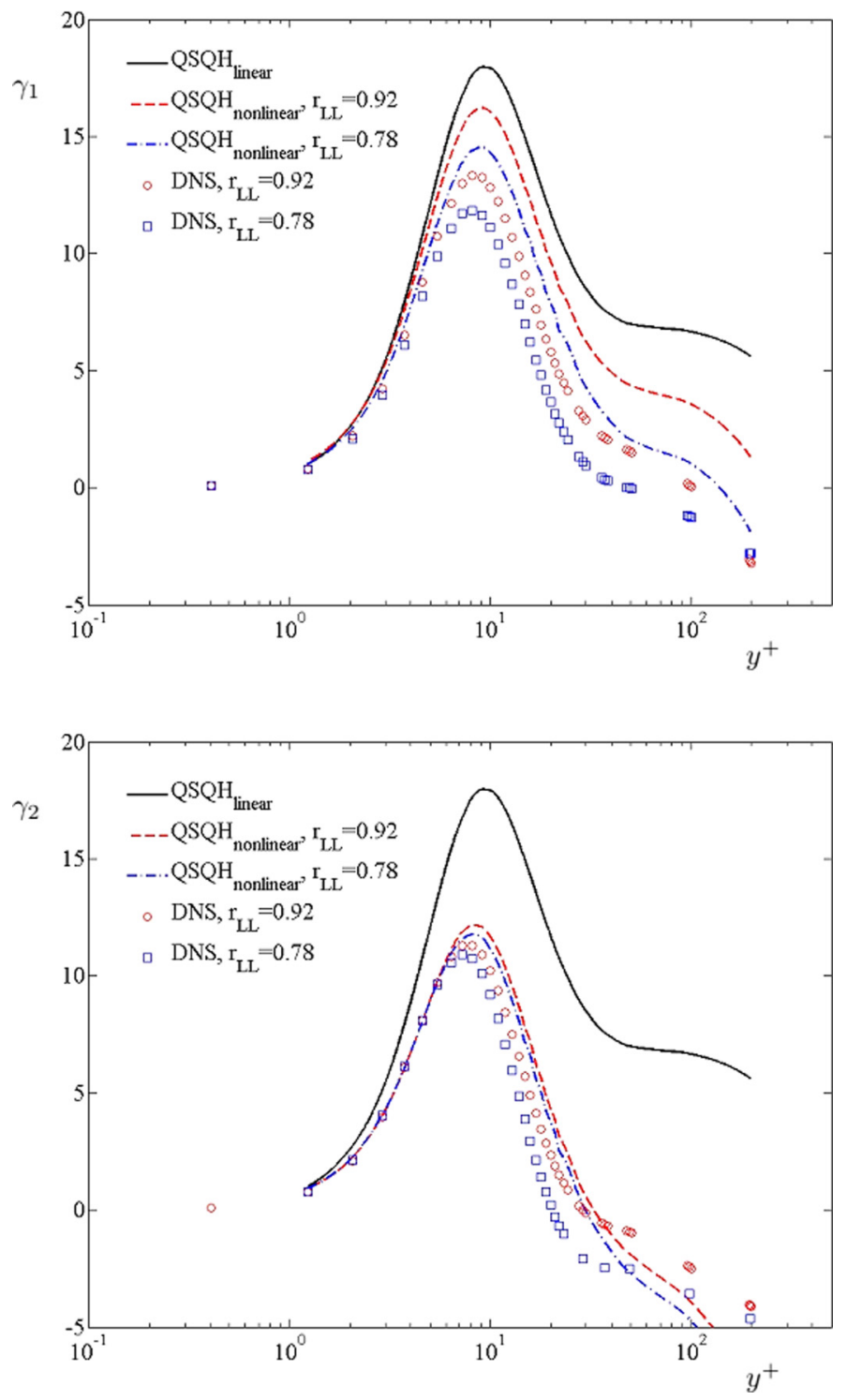

FIG. 10. The black solid curve is $\gamma$, which is the main term for $\gamma_{1}$ and $\gamma_{2}$. The red dashed curves are for the expansions including the quadratic terms for $\gamma_{1}$ and $\gamma_{2}$ calculated with the large-scale filters of $r_{L L}=0.92$ and the blue dash-dotted curves are for those of $r_{L L}=0.78$. Red circles and blue square are Eq. (24) calculated from DNS with the large-scale filters of $r_{L L}=0.92$ and $r_{L L}=0.78$, respectively.

of the QSQH mechanism of Fig. 11 and the observations. Namely, the observations show that the distance from the wall to the correlation reversal point, expressed in wall units, increases quite significantly with Re increasing (see, for example, Fig. 4 in Ref. [35]). The applicability of a QSQH hypothesis at a large distance from the wall can be questioned. Within the quadratic QSQH theory the Re effect on $\gamma_{1}$ is reduced to the change in $\left\langle u_{\tau_{L}}^{\prime 3}\right\rangle /\left\langle u_{\tau_{L}}^{\prime 2}\right\rangle$, which depends on the filter. Hence, at this point the question of whether the observed correlation reversal can be explained within the QSQH theory remains open. 


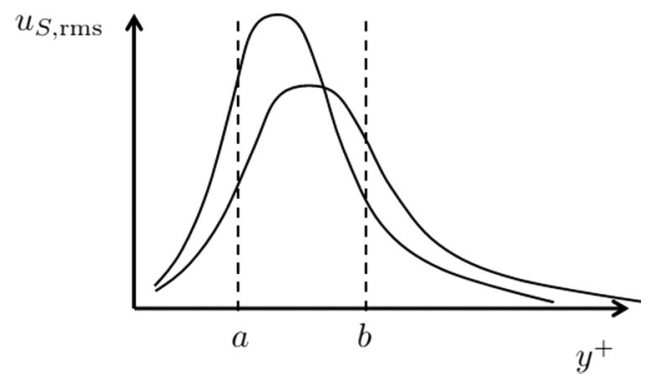

FIG. 11. Physical mechanism of the correlation reversal: The curve with the higher maximum corresponds to positive large-scale fluctuation.

An interesting question is whether another correlation reversal point will appear much closer to the wall for other Re and a different filter. Figure 1 in Ref. [31] shows the behavior consistent with such an idea, namely, there is a minimum of the correlation coefficient as a function of the wall distance near $y^{+}=30$, that is, where the slope of $\tilde{u}_{\text {rms }}(y)$ is negative. The quasisteady mechanism of Fig. 11 might provide an explanation of this minimum.

\section{E. Reynolds-number universality}

The data set used in the present study corresponds to a single value of Re. As a result, the above comparisons can test only the quasisteadiness hypothesis, but not the hypothesis that the statistical properties of $\tilde{u}(\tilde{t}, \tilde{x}, \tilde{y}, \tilde{z})$ are independent of Re. An attempt to test the second hypothesis can be made on the basis of the data on $\tilde{u}_{\mathrm{rms}}$ available in the literature. However, applying Eq. (17) requires the knowledge of $\left\langle u_{\tau_{L}}^{\prime 2}\right\rangle$, which is not available for such data. This difficulty can be circumvented by subtracting the values of $u_{\mathrm{rms}}^{2}$ for two different Re. Neglecting the second term in the square brackets in Eq. (17), which we know is justified, taking a logarithm, taking a derivative, and multiplying by $y$ then gives

$$
D=y \frac{d}{d y} \ln \left(\left.u_{\mathrm{rms}}^{2}\right|_{\mathrm{Re}_{1}}-\left.u_{\mathrm{rms}}^{2}\right|_{\mathrm{Re}_{2}}\right) \approx y \frac{d}{d y} \ln \left(\tilde{U}(y)+y \frac{d \tilde{U}}{d y}\right)^{2} .
$$

Multiplying by $y$ is desirable because otherwise the quantity would tend to infinity as $y \rightarrow 0$, thus making plotting more difficult. Figure 12 shows the comparisons for the direct numerical simulation

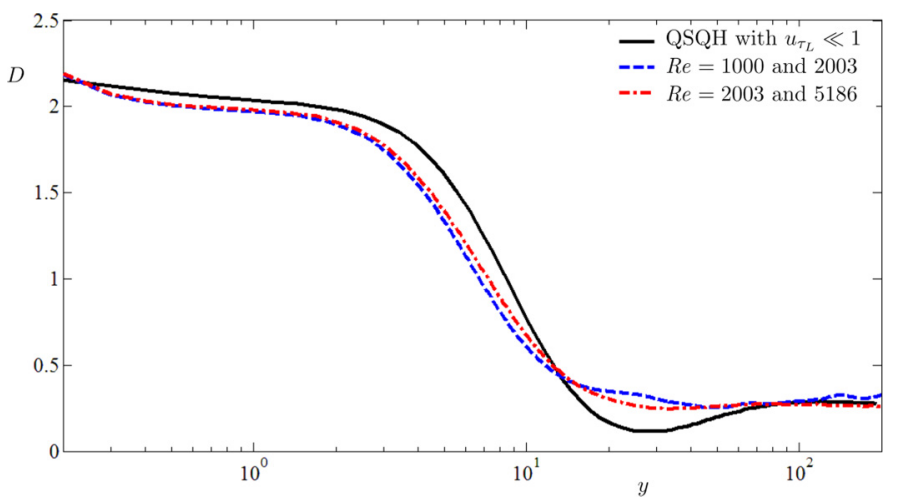

FIG. 12. Logarithmic derivative of the increment with Re of the rms fluctuation velocity, multiplied by $y$ [Eq. (30)]: the QSQH prediction (solid line), the numerical data [7] for Re increasing from 1000 to 2003 (dashed line), and the numerical data [7] for Re increasing from 2003 to 5186 (dash-dotted line). 
data of [7]. The numerical data do demonstrate Re independence, thus confirming our second hypothesis. The deviation from the QSQH theory might be due to the assumption that $u_{\tau_{L}} \ll 1$ used in deriving the right-hand side of (30) or due to the approximate nature of the QSQH hypothesis per se. While Fig. 12 is encouraging, more comparisons are desirable.

\section{DISCUSSION}

While indicating the limitations of the quasisteady quasihomogeneous hypothesis, the obtained results generally support it. Many of our results can be rearranged in such a way as to involve only the quantities that can be measured directly in a physical experiment, which express the relationships that could not be guessed or foreseen without the theory, but which are satisfied at least approximately by the real data. Recalling that $\tilde{U}(y) \approx U(y)$, the examples are (23), (26), (27), and (30) (Figs. 1, 8, 10, and 12, respectively). The relevance of the QSQH theory is also supported by the results of the previous works, in particular, by the agreement between the QSQH prediction [25,27] that $\alpha \approx \beta$ for the coefficients $\alpha$ and $\beta$ in the skin-friction equivalent of (1), the prediction of the dependence of $\alpha$ on the position of the outer probe (Fig. 2 in Ref. [27]), the collapse of the root-mean-square fluctuations in the QSQH variables [Fig. 3(a) in Ref. [6]], and the collapse of the probability density function of the velocity components (Fig. 13 in Ref. [26]) conditionally averaged over the events of large positive and large negative large-scale fluctuations. The agreements obtained are approximate, as is to be expected, but the volume of the favorable comparisons is now significant.

A QSQH hypothesis is approximate for several reasons. First, it assumes that the large scales are much larger than the small scales. However, turbulence has a continuous spectrum. For a Fourier cutoff filter the scales adjacent to the cutoff are close to each other. One solution [36] would be to partition the velocity into the sum of three components, large, small, and intermediate, but then the influence of the intermediate scales on the small scales will remain unaccounted for. Also, for finite Re even the largest scales are not infinitely larger than the small scales. Second, the QSQH hypothesis implies that the near-wall turbulence is in equilibrium with the skin friction. In fact, it adjusts itself with a certain time lag [2], roughly about 100 wall units [24], which is not small on the scale of near-wall turbulence processes. Third, the skin friction is the only parameter via which the large-scale motion affects the small scales within the QSQH hypothesis. The skin friction does not reflect the geometry of the large scales themselves. It is well known that the large-scale structures are inclined at about $12^{\circ}-14^{\circ}$. Accordingly, the QSQH theory error due to this inclination can be expected to be of the order of $y /\left(L_{x} \tan 13^{\circ}\right) \approx 5 y / L_{x}$, where $L_{x}$ is the filter cutoff length in the main flow direction; $L_{x}$ determines the size of what is assumed to be a large scale. These reasons for deviation from the QSQH hypothesis should be kept in mind when it is used for the analysis of the physical mechanisms of the flow and when considering the comparisons.

Clearly separating, as we did in Sec. II, the physical assumptions of quasisteadiness and quasihomogeneity from the corollaries that can be derived rigorously casts a certain light on the nature of the results. Imagine, for example, the following situation. A set of snapshots of the plane-channel flow data was collected in several experiments. In each experiment the flow parameters such as the mass flow rate, viscosity, and all other were kept constant until all the transients passed away, after which the data were recorded over a period long enough for time averaging to be accurate. Then the data from all the experiments were put together. Imagine also that the classical universality hypothesis holds. Imagine, however, that even though the parameters were kept constant during each of the experiments, they varied from experiment to experiment, but the researcher analyzing the complete set of data does not know this. It is easy to see that if the filtering operation is equivalent to time averaging in each separate experiment then all the requirements of Sec. II are satisfied exactly. Then, all the results obtained here apply and, for such a case, are rigorous. Moreover, since the empirical model of Hutchins, Marusic, and Mathis (1) is close to the linearized version of the QSQH theory, it also applies to this imaginary situation. The important observation from this is that confirmations of (1) as well as confirmations of the QSQH theory cannot be considered as confirmations of the existence of large-scale-small-scale interaction: Other interpretations are 
equally possible. For example, an uncontrolled slow variation of the flow conditions in a single experiment is just a modification of the above example.

The above-mentioned inclination of the large-scale structures is one of the reasons why we did not go into more quantitative details concerning the question of the dependence of the constants of the logarithmic law on Re. The logarithmic law is expected to persist further away from the wall than the range $y^{+}<100$ outside of which we observed the QSQH theory to diverge with the actual data. However, the QSQH theory might be improved in the future. Also, if it is applied to describe the effect of slow uncontrolled variation of the flow conditions we discussed, it is precise and should be valid at any distance from the wall. Hence, it is worth pointing out that the QSQH theory, if applicable, resolves one well-known controversy in this area. On the one hand, variation of the logarithmic-law constants with Re and flow conditions, such as the pressure gradient or the wall curvature, is often discussed and at least claimed to be observed. On the other hand, the known high-Re asymptotic justification for the logarithmic law itself implies that those constants are independent of Re or other parameters. Hence, if, for example, the presence of the pressure gradient changes $\kappa$ or $B$, it should be expected also to destroy the logarithmic law itself. However, if there is no logarithmic law, $\kappa$ and $B$ are undefined and hence discussing their variation is meaningless: This is the controversy. The QSQH theory resolves it by showing that the universal values of $\kappa$ and $B$ are pertinent to the QSQH universal field $\tilde{u}$ and not to the actual velocity field $u$. Then, by accident or due to some deep reasons yet beyond our understanding, the main QSQH relation (5) between these two fields happens to be such that the velocity field $u$ inherits the logarithmic law for $U=\langle u\rangle$ from $\tilde{u}$, but with constants that are not universal. Thus, the QSQH theory explains logically how it can be possible to have a universal logarithmic law with nonuniversal constants, resolving the controversy.

Our experience showed that formalizing properties of the ideal filter (see Sec. II B) has the additional benefit of making derivations much easier. This happens because at many derivation steps a straightforward application of formal rules replaces the difficult decisions of what can and what cannot be neglected on the basis of the intuitive assumption that the large scales are much larger than the small scales.

Another observation is that the physical assumptions of Sec. II do not include anything concerning the inner-outer interactions. As far as the present results are concerned, the QSQH hypothesis might as well be approximately valid because the large outer structures imprint themselves onto the near-wall turbulence or because the near-wall turbulence generates both large scale and small scales, which might or might not imprint themselves on the outer-layer structures. Determining whether there is a causality relationship between the outer structures and the inner structures, and if yes, which is the cause and which is the effect, requires an analysis beyond the QSQH theory. The aforementioned time lag, for example, might indicate that the large-scale skin-friction fluctuations are caused by the outer structures rather than being the feature of the near-wall processes.

Note that while the early works on the large-small scale interaction often discussed the superposition effect, this effect is absent from the present formulation of the QSQH hypothesis (5). The expression (20) for the coefficient $\alpha(y)$, often termed the superposition coefficient, shows that it is the combination of the amplitude modulation (the term with $\tilde{U}$ ) and the scale modulation (the term with $d \tilde{U} / d y)$ disguised by the linearization. The modulation coefficient $\beta(y)$ also includes a contribution from the scale-modulation effect.

According to (12) the instantaneous values of the large-scale velocity fluctuations depend only on the large-scale skin friction and the mean universal profile $\tilde{U}(y)$, but do not depend on the other details of the universal function, while the small-scale fluctuations (14) are in fact an amplitude and frequency or scale modulated universal fluctuation in a pure form. In this sense, the universal velocity distribution is a small-scale velocity distribution.

Many of the scale-interaction studies use the technique of conditional averaging with the condition of the fluctuation being above or below a certain threshold, as in Refs. [6] or [26]. This is often dictated by what data are contained in the database. The significant and subtle role played by the nonlinearity of the dependence on the amplitude of large scales in the phenomenon of the 
modulation shows that such conditional averaging might be too crude a tool. Ideally, the direct numerical simulation database should contain enough data for determining the statistical properties of the universal function in the QSQH hypothesis (5) in spite of the scale modulation effect distorting the grid layers in the universal coordinates.

The bulk of our comparisons refer to a single value of Re. Thus, our results establish, to the degree of accuracy of our comparisons, only the validity of the QSQH hypothesis per se. As far as the Re independence is concerned, apart from Fig. 12, we can rely only on the universality tests for (1) conducted by Marusic and co-workers in the numerous studies. More analysis is required to establish the Re independence for the features that go beyond (1). Another important question for future studies is the selection of the filter. Even though the filter we used is on the Pareto front, the selection of the particular point on this front remains somewhat arbitrary.

\section{CONCLUSION}

In the present work we gave a formal axiomatic statement of the hypothesis of the quasisteady quasihomogeneous nature of the interaction between the large and small scales in the near-wall part of turbulent flows, separating the physical assumptions from the rigorous derivations. This makes the derivations easier and allows better insight into the nature of the hypothesis.

The method was proposed of finding the optimal large-scale filter by multiobjective optimization, with the two objectives being a large correlation between large-scale fluctuations near the wall and in the layer at a certain finite distance from the wall and a small correlation between the small scales in the same layers. The filter was demonstrated to give good results within the framework of quasisteady quasihomogeneous theory.

Within the thus-introduced quasisteady quasihomogeneous theory, expansions for various quantities were found up to the second order of magnitude with respect to the amplitude of the large-scale fluctuations. Including the nonlinear effects improved the agreement between the theory and the numerical data. Full account for nonlinearity might give further improvements.

The results obtained leave open the question whether the reversal of the correlation between the large-scale velocity and the fluctuation intensity can be explained on the basis of the quasisteady quasihomogeneous hypothesis. The quasisteady quasihomogeneous theory was shown to imply a dependence of the mean-velocity logarithmic-law constants on Re, but no quantitative comparisons were made. The main overall result of the present work is the demonstration of the relevance and the further development of the quasisteady quasihomogeneous theory for near-wall turbulent flows.

\section{ACKNOWLEDGMENTS}

The authors are grateful to Professor M. Leschziner, Dr. E. Touber, and Dr. L. Agostini for providing access to the results of the direct numerical simulation they conducted and for numerous discussions and to Professor B. Ganapathisubramani and Dr. N. Hutchins for the useful discussion of an early draft of the paper.

[1] K. N. Rao, R. Narasimha, and M. A. Narayanan, The bursting phenomenon in a turbulent boundary layer, J. Fluid Mech. 48, 339 (1971).

[2] P. R. Bandyopadhyay and A. K. M. F. Hussain, The coupling between scales in shear flows, Phys. Fluids 27, 2221 (1984).

[3] G. L. Brown and A. S. W. Thomas, Large structure in a turbulent boundary layer, Phys. Fluids 20, S243 (1977).

[4] I. Marusic, B. J. McKeon, P. A. Monkewitz, H. M. Nagib, A. J. Smits, and K. R. Sreenivasan, Wall-bounded turbulent flows at high Reynolds numbers: Recent advances and key issues, Phys. Fluids 22, 065103 (2010). 
[5] A. J. Smits, B. J. McKeon, and I. Marusic, High Reynolds number wall turbulence, Ann. Rev. Fluid Mech. 43, 353 (2011).

[6] J. Jiménez, Cascades in wall-bounded turbulence, Annu. Rev. Fluid Mech. 44, 27 (2012).

[7] M. Lee and R. D. Moser, Direct numerical simulation of turbulent channel flow up to $\operatorname{Re}_{\tau}=5200$, J. Fluid Mech. 774, 395 (2015).

[8] M. A. Leschziner, H. Choi, and K.-S. Choi, Flow-control approaches to drag reduction in aerodynamics: Progress and prospects, Philos. Trans. R. Soc. London A 369, 1349 (2011).

[9] Ricardo García-Mayoral and Javier Jiménez, Drag reduction by riblets, Philos. Trans. R. Soc. London A 369, 1412 (2011).

[10] P. Luchini, Reducing the turbulent skin friction, in Computational Methods in Applied Sciences '96, edited by J.-A. Désidéri et al. (Wiley, Chichester, 1996), pp. 466-470.

[11] P. R. Spalart and J. D. McLean, Drag reduction: Enticing turbulence, and then an industry, Philos. Trans. R. Soc. London A 369, 1556 (2011).

[12] J. Kim, Physics and control of wall turbulence for drag reduction, Philos. Trans. R. Soc. London A 369, 1396 (2011).

[13] K.-S. Choi, T. Jukes, and R. Whalley, Turbulent boundary-layer control with plasma actuators, Philos. Trans. R. Soc. London A 369, 1443 (2011).

[14] M. Quadrio, Drag reduction in turbulent boundary layers by in-plane wall motion, Philos. Trans. R. Soc. London A 369, 1428 (2011).

[15] O. Blesbois, S. I. Chernyshenko, E. Touber, and M. A. Leschziner, Pattern prediction by linear analysis of turbulent flow with drag reduction by wall oscillation, J. Fluid Mech. 724, 607 (2013).

[16] C. A. Duque-Daza, M. F. Baig, D. A. Lockerby, S. I. Chernyshenko, and C. Davies, Modeling turbulent skin-friction control using linearized Navier-Stokes equations, J. Fluid Mech. 702, 403 (2012).

[17] R. Moarref and M. R. Jovanović, Model-based design of transverse wall oscillations for turbulent drag reduction, J. Fluid Mech. 707, 205 (2012).

[18] S. Chernyshenko, Drag reduction by a solid wall emulating spanwise oscillations. Part 1, arXiv:1304.4638.

[19] E. Hurst, Q. Yang, and M. Yongmann, The effect of Reynolds number on turbulent drag reduction by streamwise traveling waves, J. Fluid Mech. 759, 28 (2014).

[20] S. Chernyshenko, Turbulent drag reduction: An overview of theoretical research at the Department of Aeronautics of Imperial College London, Joint ERCOFTAC-PLASMAERO Workshop, Toulouse, 2012, http://www.ercoftac.org/downloads/sig20/workshop_book_of_abstracts.pdf (unpublished).

[21] N. Hutchins and I. Marusic, Large-scale influences in near-wall turbulence, Philos. Trans. R. Soc. London A 365, 647 (2007).

[22] I. Marusic, R. Mathis, and N. Hutchins, Predictive model for wall-bounded turbulent flow, Science 329, 193 (2010).

[23] R. Mathis, N. Hutchins, and I. Marusic, A predictive inner-outer model for streamwise turbulence statistics in wall-bounded flows, J. Fluid Mech. 681, 537 (2011).

[24] B. Ganapathisubramani, N. Hutchins, J. P. Monty, D. Chung, and I. Marusic, Amplitude and frequency modulation in wall turbulence, J. Fluid Mech. 712, 61 (2012).

[25] R. Mathis, I. Marusic, S. I. Chernyshenko, and N. Hutchins, Estimating wall-shear-stress fluctuations given an outer region input, J. Fluid Mech. 715, 163 (2013).

[26] L. Agostini and M. A. Leschziner, On the influence of outer large-scale structures on near-wall turbulence in channel flow, Phys. Fluids 26, 075107 (2014).

[27] S. Chernyshenko, I. Marusic, and R. Mathis, Quasi-steady description of modulation effects in wall turbulence, arXiv:1203.3714.

[28] J. Kevorkian and J. D. Cole, Perturbation Methods in Applied Mathematics, Applied Mathematical Sciences Vol. 34 (Springer, New York, 2013).

[29] P. Schlatter and R. Örlü, Quantifying the interaction between large and small scales in wall-bounded turbulent flows: A note of caution, Phys. Fluids 22, 051704 (2010).

[30] M. Bernardini and S. Pirozzoli, Inner/outer layer interactions in turbulent boundary layers: A refined measure for the large-scale amplitude modulation mechanism, Phys. Fluids 23, 061701 (2011). 
[31] R. Mathis, I. Marusic, N. Hutchins, and K. R. Sreenivasan, The relationship between the velocity skewness and the amplitude modulation of the small scale by the large scale in turbulent boundary layers, Phys. Fluids 23, 121702 (2011).

[32] S. Duvvuri and B. J. McKeon, Triadic scale interactions in a turbulent boundary layer, J. Fluid Mech. 767, R4 (2015).

[33] L. Agostini, E. Touber, and M. A. Leschziner, Spanwise oscillatory wall motion in channel flow: Drag-reduction mechanisms inferred from DNS-predicted phase-wise property variations at $\operatorname{Re}_{\tau}=1000$, J. Fluid Mech. 743, 606 (2014).

[34] R. Mathis, N. Hutchins, and I. Marusic, Large-scale amplitude modulation of the small-scale structures in turbulent boundary layers, J. Fluid Mech. 628, 311 (2009).

[35] D. Chung and B. J. McKeon, Large-eddy simulation of large-scale structures in long channel flow, J. Fluid Mech. 661, 341 (2010).

[36] O. R. H. Buxton, Modulation of the velocity gradient tensor by concurrent large-scale velocity fluctuations in a turbulent mixing layer, J. Fluid Mech. 777, R1 (2015). 